\title{
Screening and Validation of p38 MAPK Involved in Ovarian Development of Brachymystax lenok
}

\begin{abstract}
Tianqing Huang ${ }^{1}$, Wei Gu ${ }^{1}$, Enhui Liu ${ }^{1}$, Lanlan Zhang ${ }^{2}$, Fulin Dong ${ }^{1}$, Xianchen $\mathrm{He}^{3}$, Wenlong Jiao ${ }^{4}$, Chunyu $\mathrm{Li}^{5}$, Bingqian Wang ${ }^{1 *}$ and Gefeng $\mathrm{Xu}{ }^{1 *}$

${ }^{1}$ Key Laboratory of Freshwater Aquatic Biotechnology and Breeding, Ministry of Agriculture and Rural Affairs, Heilongjiang River Fisheries Research Institute, Chinese Academy of Fishery Sciences, Harbin, China, ${ }^{2}$ Heilongjiang Province General Station of Aquatic Technology Promotion, Harbin, China, ${ }^{3}$ Heilongjiang Aquatic Animal Resource Conservation Center, Harbin, China, ${ }^{4}$ Gansu Fisheries Research Institute, Lanzhou, China, ${ }^{5}$ Xinjiang Tianyun Organic Agriculture Co., Yili Group, Hohhot, China
\end{abstract}

Brachymystax lenok (lenok) is a rare cold-water fish native to China that is of high meat quality. Its wild population has declined sharply in recent years, and therefore, exploring the molecular mechanisms underlying the development and reproduction of lenoks for the purposes of artificial breeding and genetic improvement is necessary.

Edited by:

Roger Huerlimann,

Okinawa Institute of Science and

Technology Graduate

University, Japan

Reviewed by:

Xiangshan Ji.

Shandong Agricultural

University, China

Jake Goodall,

University of Iceland, Iceland

*Correspondence:

Bingqian Wang

wangbingqian@hrfri.ac.cn

Gefeng Xu

xugefeng@hrfri.ac.cn

Specialty section:

This article was submitted to

Livestock Genomics,

a section of the journal

Frontiers in Veterinary Science

Received: 27 August 2021

Accepted: 13 January 2022

Published: 16 February 2022

Citation:

Huang T, Gu W, Liu E, Zhang L, Dong F, He X, Jiao W, Li C, Wang B and XU G (2022) Screening and Validation of p38 MAPK Involved in

Ovarian Development of

Brachymystax lenok.

Front. Vet. Sci. 9:752521

doi: 10.3389/fvets.2022.752521
The lenok comparative transcriptome was analyzed by combining single molecule, real-time, and next generation sequencing (NGS) technology. Differentially expressed genes (DEGs) were identified in five tissues (head kidney, spleen, liver, muscle, and gonad) between immature [300 days post-hatching (dph)] and mature [three years posthatching (ph)] lenoks. In total, 234,124 and 229,008 full-length non-chimeric reads were obtained from the immature and mature sequencing data, respectively. After NGS correction, 61,405 and 59,372 non-redundant transcripts were obtained for the expression level and pathway enrichment analyses, respectively. Compared with the mature group, 719 genes with significantly increased expression and 1,727 genes with significantly decreased expression in all five tissues were found in the immature group. Furthermore, DEGs and pathways involved in the endocrine system and gonadal development were identified, and p38 mitogen-activated protein kinases (MAPKs) were identified as potentially regulating gonadal development in lenok. Inhibiting the activity of p38 MAPKs resulted in abnormal levels of gonadotropin-releasing hormone, follicle -stimulating hormone, and estradiol, and affected follicular development. The full-length transcriptome data obtained in this study may provide a valuable reference for the study of gene function, gene expression, and evolutionary relationships in B. lenok and may illustrate the basic regulatory mechanism of ovarian development in teleosts.

Keywords: full-length transcriptome, p38 MAPK, ovarian development, Brachymystax lenok, SMRT and NGS

\section{INTRODUCTION}

Brachymystax lenok (lenok) is an economically important fish in the Amur Basin with highquality nutrient-rich meat. As a rare cold water fish native to China, lenok has been listed as a vulnerable species in the Red Book of Endangered Animals in China because of its sharp decline in the wild, and is classified as aquatic wildlife under second-class protection (1). Recent studies 
on lenok have mainly focused on resource investigation, nutritional physiology, and artificial reproduction (2-4), and studies on the genetic analysis and reproductive regulation mechanisms of lenok are limited because of sparse genetic information. It is important to analyse the molecular mechanisms that regulate lenok reproduction and development to facilitate better breeding and genetic improvement.

The analysis of full-length transcriptome can demonstrate the type and number of genes at the molecular level and is an effective method for revealing the regulatory mechanisms of different physiological and biochemical processes. Many nonmodel organisms lack reference genome sequence information, and full-length transcriptome sequencing is a rapid and effective method for investigating gene expression, gene function, and evolutionary analysis in this species (5-7). Single molecule real-time sequencing technology (SMRT) based on the Pacific Biosciences (PacBio) platform has the benefits of long reading fragments and high accuracy. Full-length mRNA can be generated directly without assembly, and this technique has been successfully used for the full-length transcriptome analysis of multiple species, such as cattle (8), rabbits (9), mice (10), and shrimp (11). It has also been widely used in teleosts (12-14).

Next generation sequencing based on the Illumina platform is helpful for characterizing various biological processes and exploiting underlying gene activity. Over the past decade, numerous studies have been conducted on genetic and developmental transcriptomics in fish, including microarray and the serial analysis of gene expression. In zebrafish, transcriptome data at nine different stages of embryonic development were comprehensively analyzed to identify the key roles of pathways and functional genes involved in development (15). In haddock (Atlantic Haddock), a genetic network for development has been established by analyzing transcriptome data from the embryo to the early developmental stages of juveniles (16). Numerous studies on gonadal development have been conducted using transcriptome sequencing. For example, genes that are differentially expressed during testis development have been characterized in the channel catfish (Ictalurus punctatus) (17). In the spotted knifejaw (Oplegnathus punctatus) and fugu (Takifugu rubripes), a large number of differential genes were identified in the testes of adult fish compared to the ovaries $(18,19)$. In gonads from 3-24 weeks after the fertilization (immature to mature) of zebrafish, the dynamic trend of miRNA abundance was characterized by miRNA sequencing (20). However, the number of studies on gonadal development using full length transcriptome methods is limited.

The collection of gonadal tissue samples from lenok could only commence at 300 days post-hatching (dph), and lenok at three years post-hatching ( $\mathrm{ph}$ ) were on the verge of ovulation. These two periods are representative of immature and mature groups for transcriptome sequencing. PacBio and Illumina sequencing were combined to generate two complete full length transcriptomes of immature and mature lenok by analyzing gene expression in five different tissues (liver, muscle, spleen, head kidney, and gonad) and screening for DEGs related to lenok ovarian development. p38 mitogen-activated protein kinases (MAPKs) were defined to express the most significant difference between the immature and mature gonads. MAPKs are a form of conserved serine/threonine protein kinases that participate in the regulation of multiple physiological functions in the form of intracellular signal transmission, and are an important signal transduction system in cells (21). Furthermore, p38 MAPK has been suggested as playing an important role in lenok development and reproduction. As one of the important members of the MAPK family, it is widely expressed in the thyroid, testis, ovary, and pituitary tissues of mammals (22), and plays an important role in the reproductive process (23). However, the role of p38 MAPK in lenok is currently unknown. Therefore, p38 MAPK was selected to demonstrate its role in the balance of reproductive endocrine hormones and the follicular development of lenok. The full-length transcriptome data obtained in this study can provide a valuable reference for further studies on the mechanism of gonadal development and maturation, and make an important contribution to researching the genetic improvement of lenok.

\section{MATERIALS AND METHODS}

\section{Ethics Statement}

All experiments were performed in accordance with the European Communities Council Directive (86/609/EEC). The experiments were approved by the Animal Husbandry Department of the Heilongjiang Animal Care and Use Committee (202110384464). All fish involved in this research were bred following the guidelines of the Animal Husbandry Department of Heilongjiang, China.

\section{Fish Sampling and RNA Purification}

The gonad $(\mathrm{G})$, head kidney $(\mathrm{K})$, liver (L), muscle $(\mathrm{M})$, and spleen (S) were collected from three mature and 12 immature samples of $B$. lenok, which were bred at the Bohai experimental station of the Heilongjiang River Fisheries Research Institute $\left(129^{\circ} 04^{\prime} 64.7753^{\prime \prime} \mathrm{E} ; 44^{\circ} 14^{\prime} 5.983^{\prime \prime} \mathrm{N}\right)$. All samples used in this experiment were obtained from female lenok. The immature group was $300 \mathrm{dph}$ and the mature group was three years ph. Four immature tissue samples were mixed into one RNA sample, which required three repeat RNA samples for sequencing. Therefore, the immature sample size was 12. However, one mature tissue sample can constitute one RNA sample and three duplicate samples require a mature sample size of $N=3$. Before tissue collection, the fish were euthanized with an overdose of anesthesia in MS-222, as reported previously (2). Each tissue sample was immediately placed into $2 \mathrm{~mL}$ sterile tubes and placed in liquid nitrogen. After storing for $1 \mathrm{~h}$, all samples were transferred to a $-80{ }^{\circ} \mathrm{C}$ refrigerator for further analysis. In addition, gonad samples at $300 \mathrm{dph}, 750 \mathrm{dph}$, and three years ph were used for western blot analysis and were kept at $-20^{\circ} \mathrm{C}$. TRIzol reagent (Invitrogen, CA, USA) was used to extract the total RNA, and only RNA samples with a RIN number greater than 7.0 were kept for subsequent experiments. One microgram of each RNA sample was pooled and sequenced using PacBio single-molecule, long-read sequencing (PacBio Sequel, Menlo Park, USA), and Illumina sequencing (Illumina NovaSeq 6000, 
California, USA) in parallel. The correlation of each sample was R2 $>0.8$ (Supplementary Figure S1).

\section{Complementary DNA (CDNA) Library Construction and PacBio Sequencing}

The SMARTer PCR cDNA Synthesis Kit (Takara Bio USA, Inc.) was used to prepare the PacBio cDNA library using the following steps: Mix tube 1 ( $2 \mu \mathrm{L}$ total RNA, $1 \mu \mathrm{L}$ 3'SMART CDS Primer II $\mathrm{A}$, and $1.5 \mu \mathrm{L}$ deionized $\mathrm{H}_{2} \mathrm{O}$ ) at $72{ }^{\circ} \mathrm{C}$ for $3 \mathrm{~min}$, and $42{ }^{\circ} \mathrm{C}$ for $2 \mathrm{~min}$. Then, mix tube $2(2 \mu \mathrm{l}$ 5xFirst-Strand Buffer, $0.25 \mu \mathrm{l}$ DTT, $1 \mu \mathrm{l}$ dNTP Mix, $1 \mu \mathrm{l}$ SMARTer II A Oligonucleotide, $0.25 \mu \mathrm{l}$ RNase Inhibitor, and $1 \mu$ l SMART Scribe Reverse Transcriptase), and then add into tube 1 at $42^{\circ} \mathrm{C}$ for $1 \mathrm{~h}$, and $72^{\circ} \mathrm{C}$ for $10 \mathrm{~min}$. The full-length cDNA was subjected to PCR amplification. The quality and concentration of the cDNA library were determined using a Qubit 2.0 Fluorometer and an Agilent 2100 bioanalyzer (24). The 1-6 KB library was sequenced using PacBio Sequel.

\section{CDNA Library Preparation for Illumina Sequencing}

Three replicates for each of the five tissues (gonad, head kidney, liver, muscle and spleen), making a total of 15 RNA samples, were fragmented into small pieces at high temperatures. The mRNA-Seq sample preparation kit (Illumina, San Diego, CA, USA) was used for the reverse transcription of RNA fragments to construct the final cDNA library, and fragments of 250-300 bp were selected using AMPure XP beads (25). The final cDNA library was assessed by PCR and the quality of the cDNA library was determined using an Agilent 2100 Bioanalyzer. Pairedend sequencing was performed on an Illumina NovaSeq 6000, following the recommended protocol (26).

\section{Data Analysis of PacBio Sequencing}

Raw reads were processed into error-corrected reads of inserts (ROIs) using the Iso-seq pipeline (27) with min full pass $=3$ and $\min$ predicted accuracy $=0.9$. Full-length, non-chimeric (FLNC) transcripts were determined by searching for the polyA tail signal and the $5^{\prime}$ and $3^{\prime} \mathrm{cDNA}$ primers in the ROIs (28). Iterative clustering for error correction (ICE) was used to obtain consensus isoforms, and FL consensus sequences from the ICE were polished using Quiver (29). High-quality FL transcripts were classified with a post-correction accuracy above $99 \%$. The cluster database with high identity and tolerance (CD-HIT, http://weizhongli-lab.org/cd-hit/) was used for IsoSeq high-quality FL transcripts to remove redundancy (identity $>0.99$ ).

\section{Data Analysis of Illumina Sequencing}

Raw reads in Fastq format were first processed using Perl scripts from our laboratory. In this step, clean reads were obtained by removing reads containing adapters, reads containing poly$\mathrm{N}$, and low-quality reads from the raw data. The Q20, Q30, GC content, and sequence duplication levels of the clean data were concurrently calculated. All downstream analyses were based on high quality clean data. These clean reads were then mapped to the PacBio reference genome sequence. Only reads with a perfect match or one mismatch were further analyzed and annotated based on the reference genome. Hisat2 (30) tools were used to map the reference genome. Gene expression levels were estimated by fragments per kilobase of transcript per million fragments mapped. Prior to differential gene expression analysis, for each sequenced library, the read counts were adjusted using the edgeR (31) program package through one scaling normalized factor. A differential expression analysis of the two samples was performed using the EBSeq R (32) package. The resulting false discovery rate was adjusted using the posterior probability of $\mathrm{DE}$ (PPDE). A false discovery rate $<0.01$, and fold change $\geq 2$ were set as thresholds for significantly differential expression.

\section{Structure Analysis}

Simple sequence repeats (SSRs) in the transcriptome were identified using MISA (http://pgrc.ipk-gatersleben.de/misa/). Candidate coding regions within the transcript sequences were identified using TransDecoder (https://github.com/ TransDecoder/TransDecoder/releases). Iso-Seq ${ }^{\mathrm{TM}}$ data were directly used to run all-vs-all BLAST (33) with high identity settings. BLAST alignments that met all criteria were considered products of candidate AS events. The coding potential calculator (CPC, http://cpc2.cbi.pku.edu.cn), coding non-coding index (CNCI, https://github.com/www-bioinfo-org/CNCI), coding potential assessment tool (CPAT, http://rna-cpat.sourceforge. net/), and protein family database (Pfam, http://pfam.xfam.org/) were combined to sort non-protein-coding RNA candidates from putative protein-coding RNAs in the transcripts. Transcripts with lengths greater than $200 \mathrm{nt}$ and having more than two exons were selected as long non-coding RNA (lncRNA) candidates.

\section{Gene Functional Annotation and Enrichment Analysis}

Gene function was annotated based on the following databases: NCBI non-redundant protein sequences (NR, http://www. ncbi.nlm.nih.gov/), protein family (Pfam, http://pfam. xfam.org/), Clusters of Orthologous Groups of proteins (KOG/COG/eggNOG, http://www.ncbi.nlm.nih.gov/COG/), Swiss-Prot (a manually annotated and reviewed protein sequence database, http://www.uniprot.org/), Kyoto Encyclopedia of Genes and Genomes (KEGG, http://www.genome.jp/kegg/), and Gene Ontology (GO). The GO enrichment analysis of differentially expressed genes (DEGs) was implemented using the GOseq R (34) packages based on Wallenius non-central hyper-geometric distribution (34), which can adjust for gene length bias in DEGs. KOBAS (35) software was used to test the statistical enrichment of DEGs in KEGG pathways.

\section{Validation of Expressions of DEGs by Real-Time PCR}

Real-time PCR was performed to validate the RNAs involved in RNA-Seq. The same RNA samples were used for the deep sequencing. Twelve mRNAs were detected using real-time PCR. A PrimeScript RT Reagent Kit (TaKaRa, Shiga, Japan) was used for reverse transcription. Real-time PCR was performed using FastStart Universal SYBR ${ }^{\circledR}$ Green Master Mix (Roche, Switzerland) and a CFX96 C1000 touch thermal cycler (BioRad, USA). Beta-actin was used as the reference gene. The Ct 
values were measured, and the value of the target sequence normalized to the reference sequence was calculated as $2^{-\Delta \Delta} \mathrm{Ct}$. The statistical analysis was performed using SPSS (36) version 13.0 for Microsoft Windows.

\section{Western Blot Analysis}

Proteins were extracted from gonad samples at $300 \mathrm{dph}$ (immature, $n=3$ ) and three years post-hatching (mature, $n=$ 3) of female lenoks that were reared under identical conditions to individuals used in transcriptome analyses. To explore the expression profiles of p38 MAPK protein in different tissues, the proteins of the gill, heart, liver, spleen, intestine, skin, and ovary tissues were extracted from female lenok at $750 \mathrm{dph}$ $(n=3)$, which was a time of rapid follicular development. The proteins were boiled for $20 \mathrm{~min}$ in SDS-PAGE loading buffer and separated using 12\% SDS-PAGE gels. The proteins were transferred to polyvinylidene fluoride membranes (Bio-Rad, USA) and analyzed by western blotting. Anti-p38 MAPK (bs0637R, Bioss, 1:10000), anti-phospho-p38 MAPK (Thr180) (bs5476R, Bioss, 1:5000), and beita-actin (ym3121, Immunoway, 1:5000) were used as primary antibodies, and peroxidaseconjugated AffiniPure goat anti-rabbit IgG (1:2000) was used as the secondary antibody (Cell Signaling Technology, USA). After washing with PBST, the protein bands were visualized by infrared fluorescence using the Odyssey Imaging System (LI-COR InC).

\section{SB203580 Inhibitor Injection}

SB203580 (Biorbyt) was used to inhibit the phosphorylation of p38 MAPK. Lenoks of approximately $750 \mathrm{dph}$ were randomly divided into three groups $(n=3)$. CK was the notreatment group, the negative control (DMSO) was injected intraperitoneally with $1 \mathrm{~mL}$ DMSO, and the experimental group $(\mathrm{DMSO}+\mathrm{I})$ was injected intraperitoneally with $5 \mathrm{mg} / \mathrm{kg}$ SB203580 dissolved in $1 \mathrm{ml}$ DMSO. Seven days post-injection, plasma and gonad samples of the three groups were collected for western blot analysis, hormone level determination, and immunohistochemical assays.

\section{Reproductive Hormone Level Assay}

Polyclonal antibodies were customized based on partial amino acid sequences of follicle-stimulating hormone (FSH), luteinizing hormone $(\mathrm{LH})$, and gonadotropin-releasing hormone 3 (GnRH3) in the lenok. Enzyme-linked immunosorbent assay (ELISA) kits (MLBIO, China) were coated with specific antibodies and developed to detect FSH, LH, and GnRH3 in the lenok. Referring to D'Cotta's method (37), an ELISA was used to verify the specificity and stability of each kit. Ten positive serum samples of each coated antibody were tested, all of which were positive, and 10 blank controls were tested, all of which were negative. The coefficient of variation between and within batches was less than $15 \%$. Standard curves for each ELISA kit are shown in Supplementary Figure S2. The ELISA experiments were performed using a microplate reader at a wavelength of $450 \mathrm{~nm}$. The levels of GnRH3, FSH, LH, and estrogen (E2) were calculated using standard curves. The plasma of three lenoks in CK, DMSO, and DMSO + I groups $(n=3)$ were detected by ELISA, and each sample was assayed three times.

\section{Immunohistochemistry}

Gonad samples from the three groups were fixed in $4 \%$ paraformaldehyde for $24 \mathrm{~h}$ at $4 \mathrm{C}$, embedded in paraplast, and sectioned at $5 \mu \mathrm{m}$ thickness. Paraffin sections were incubated with $3 \%$ hydrogen peroxide in phosphate-buffered saline for $10 \mathrm{~min}$, and then blocked with $2 \%$ bovine serum albumin and $2 \%$ goat serum for $1 \mathrm{~h}$. Sections were boiled with $0.01 \mathrm{M}$ citric acid and EDTA solution for $30 \mathrm{~min}$ for antigen recovery. The $\mathrm{p}$ p38 antibody (1:300) was applied to the sections as the primary antibody at $4{ }^{\circ} \mathrm{C}$ overnight. After washing with phosphatebuffered saline, the sections were incubated with biotinylated goat anti-rabbit secondary antibody (1:1000) for $20 \mathrm{~min}$ at $25^{\circ} \mathrm{C}$. The sections were then treated with peroxidase-conjugated streptavidin, developed with $\mathrm{DAB}$, and counterstained with haematoxylin. For the negative control, sections were not incubated with the primary antibody. For this part, the gonads of three lenoks from CK, DMSO, and DMSO+I groups $(n=3)$ were analyzed.

\section{Statistical Analysis}

A one-way analysis of variance was used to assess the differences in p38 protein levels in the gills, heart, liver, spleen, intestine, head, skin and gonad, and in gonad at $300 \mathrm{dph}, 750 \mathrm{dph}$, and three years ph. It was also used to assess the differences in reproductive hormone levels in the CK, DMSO, and DMSO+I groups. Data were shown as the mean \pm SEM. The statistical analysis was performed using SPSS version 13.0 for Microsoft Windows, and the statistical significance was set at $p<0.05$.

\section{RESULTS}

\section{Transcriptome Analysis}

\section{PacBio Iso-Seq and Bioinformatic Analysis}

The PacBio iso-sequencing of lenok was completed, the clean data of mature and immature were obtained by SMRT

TABLE 1 | Statistics of the PacBio Iso-sequencing data.

\begin{tabular}{lll}
\hline cDNA library & Mature & Immature \\
\hline cDNA size & $1-6 \mathrm{~K}$ & $1-6 \mathrm{~K}$ \\
Date size (GB) & 23.21 & 22.67 \\
CCS number & 272,337 & 271,088 \\
Read bases of CCS & $810,860,211$ & $756,750,619$ \\
Mean read length of CCS & 2,977 & 2,791 \\
Mean number of passes & 37 & 39 \\
Number of FLNC reads & 229,008 & 234,124 \\
FLNC\% & $84.09 \%$ & $86.36 \%$ \\
Number of polished & 85,270 & 89,226 \\
high-quality isoforms & & 2,196 \\
Number of polished low-quality & 2,020 & \\
isoforms & & $97.17 \%$ \\
Percent of polished & $97.25 \%$ & 59,372 \\
high-quality isoforms & & \\
CD-HIT & 61,405 & \\
\hline
\end{tabular}

GB: A unit used to measure the amount of data. KB: One thousand base pairs. 
A

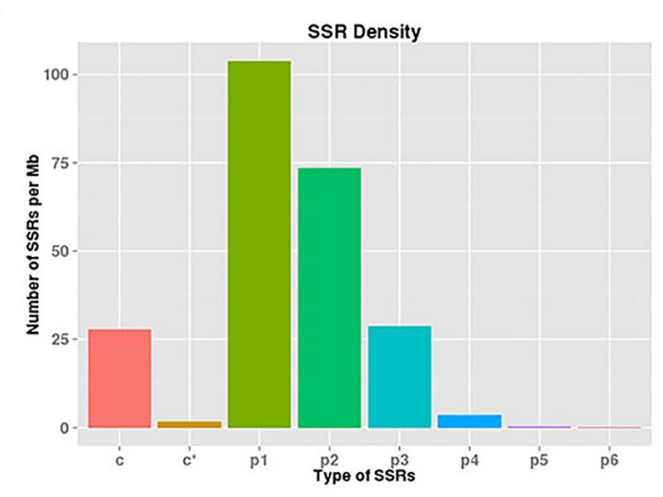

C

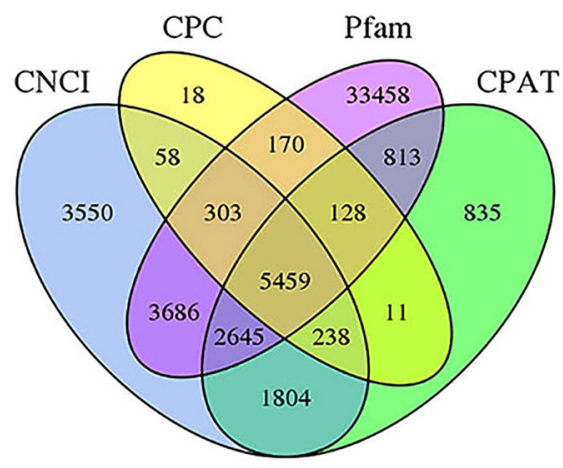

E

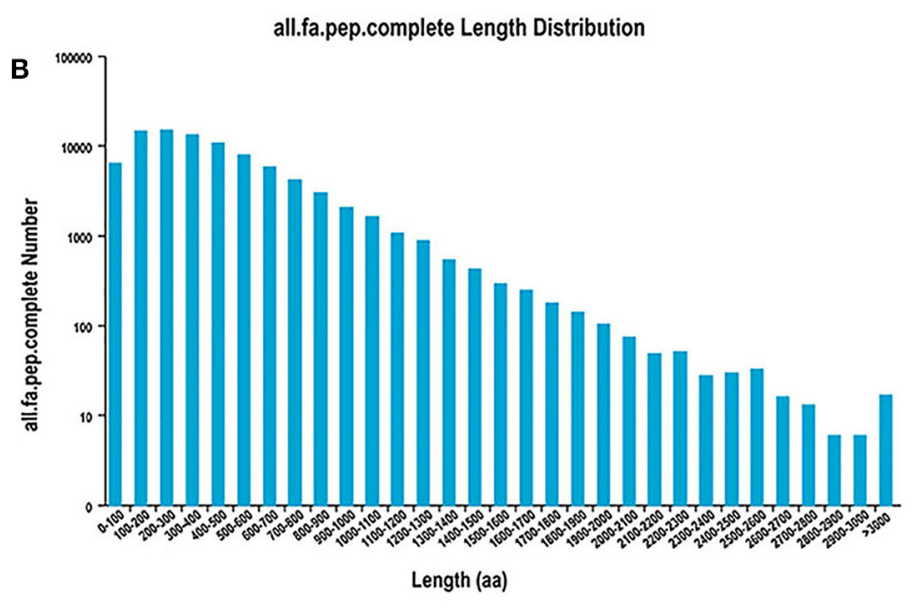

D

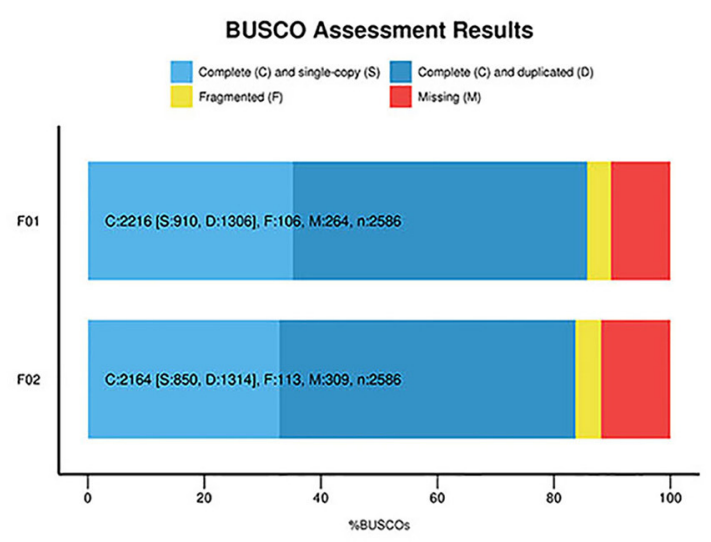

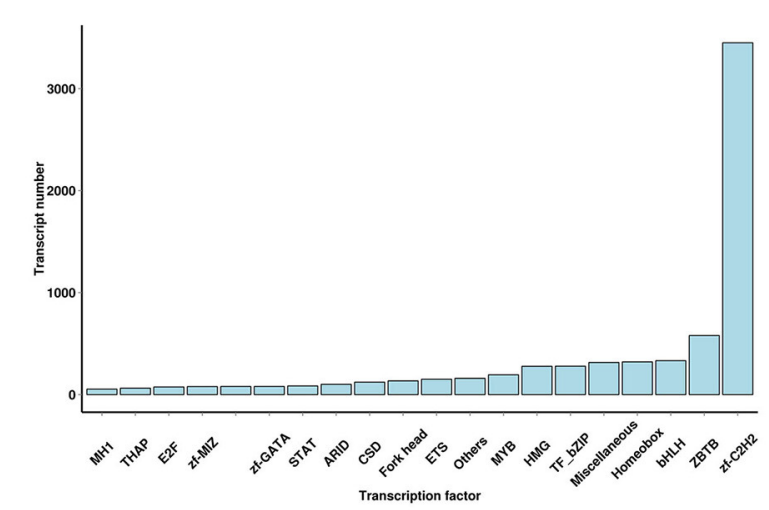

FIGURE 1 | Analysis of SSRs and identification of CDS, TFs, and IncRNAs. (A) The density of different type of SSRs; (B) The length distribution of predicted CDS; (C) Venn diagram for IncRNAs identified by four analysis methods; (D) BUSCO assessment results of the completeness of transcripts generated by CD-HIT; (E) Distribution of TF families.

sequencing technology, and the data sizes were $23.21 \mathrm{~GB}$ and 22.67 GB, respectively. Among these, 272,337 and 271,088 circular consensus sequence (CCS) reads were obtained from mature and immature samples, respectively (Table 1), with a mean length of 2,977 and $2,791 \mathrm{bp}$, respectively, using 37 and 39 passes, respectively, with full passes $\geq 3$, and a quality consensus accuracy $>0.9$. Subsequently, the CCS reads were classified as full-length non-chimeric (FLNC) with $5^{\prime}$ primer, $3^{\prime}$ primer, and poly-A and non-full length reads, with proportions of 84.09 and $86.36 \%$, respectively, for mature and immature samples. As a result, 229,008 and 234,124 high-quality FLNC reads for mature and immature samples, respectively, were obtained through the cluster of FLNC and correction. 
We obtained 85,270 and 89,226 high-quality polished consensus sequences (Table 1) for the mature and immature samples, respectively. Finally, 61,405 and 59,372 non-redundant transcripts for mature and immature samples, respectively, were obtained by removing redundant transcripts using CD-HIT. By merging the mature and immature data, we obtained 106,647 non-redundant transcripts for $B$. lenok development analysis. The PacBio Iso-Seq raw data were deposited into the SRANCBI repository. The BioProject number was PRJNA669274 and the BioProject number of next-generation sequencing was PRJNA669219.

\section{Analysis of Alternative Splicing Events, SSRs and Predictions of Coding Sequences, Transcription Factors, and LncRNAs}

In this study, 3,158 and 3,332 alternative splicing (AS) events were analyzed. The absence of a reference genome limited the identification of the AS types. For the analysis of SSRs, 104,373 sequences $(344,196,344 \mathrm{bp})$ were examined, including 103,688 SSRs and 49,478 SSR-containing sequences (Supplementary Table S1). There were 23,300 sequences containing more than one SSR, and the number of SSRs present in the compound form was 21,101 . Specifically, most sequences were mononucleotides $(42,411)$, dinucleotides $(46,227)$, and trinucleotides $(12,599)$. The number of different types of SSRs is shown in Figure 1A.

To identify putative protein-coding sequences, we predicted 100,580 open reading frames (ORFs) using the Trans-Decoder. In total, 88,856 coding sequences (CDSs) were identified with start and stop codons. The distributions of the numbers and lengths of complete CDSs are shown in Figure 1B. Among these, 14,579 transcripts $(16.41 \%)$ were distributed in the $100-200 \mathrm{bp}$ range, 14,893 transcripts $(16.76 \%)$ in the $200-300$ bp range, 13,365 transcripts (15.04\%) in the $300-400$ bp range, and 10,810 transcripts $(12.17 \%)$ in the $400-500$ bp range.

Here, 5,459 lncRNAs were predicted using a coding potential calculator (CPC), coding-non-coding index (CNCI), Pfam protein structure domain analysis, and coding potential assessment tool (CPAT) (Figure 1C) and candidate lncRNAs for future developmental research on lenok were revealed.

The completeness of the transcripts generated by CD-HIT was assessed using benchmarking universal single-copy orthologs (BUSCO, v.2.3). The results showed that 85.69 and $83.68 \%$ of the transcripts of mature and immature lenok samples, respectively, were complete (Figure 1D). Among the mature group, singlecopy and duplicated BUSCOs accounted for 41.06 and 58.94\%, respectively. In the immature group, the percentages of complete single-copy and duplicated BUSCOs were 39.28 and $60.72 \%$, respectively. Only 106 and 113 fragmented BUSCOs and 264 and 309 missing BUSCOs were found in our two databases (Figure 1D). These results all show that our database is complete and available for subsequent research.

In total, 7,628 putative transcription factors (TFs) were examined by sequencing, and the top 20 families with the highest number of TFs are shown in Figure 1E. Most TFs belonged to the zf-C2H2 (3,450), ZBTB (579), bHLH (333), homeobox (361), miscellaneous (315), TF-bZIP (279), and HMG (278) families.
TABLE 2 | Number of annotated transcripts of each database.

\begin{tabular}{ll}
\hline Annotated databases & Number of transcripts \\
\hline COG & 33,077 \\
GO & 83,729 \\
KEGG & 67,965 \\
KOG & 74,798 \\
Pfam & 88,168 \\
Swiss-Prot & 69,821 \\
eggNOG & 99,079 \\
NR & 102,107 \\
All & 102,295 \\
\hline
\end{tabular}

\section{Function Annotation of Transcripts}

The annotation information of 102,295 (95.92\%) non-redundant transcripts was obtained by blasting eight databases, namely, NR, Swiss-Prot, COG, KOG, Pfam, eggNOG, GO, and KEGG. The number of annotated transcripts in each database is listed in Table 2.

By blasting the sequences of homologous species in the NR database, $50.866(49.83 \%)$ annotated transcripts were aligned with Oncorhynchus mykiss, followed by Esox luclus (26.95\%) and Salmo salar (13.28\%) (Figure 2A).

In total, 83,729 transcripts were enriched in the three ontologies based on the GO analysis (Figure $2 \mathrm{~B}$ ). In the cellular component (CC), transcripts were mainly enriched in cells $(44,531)$, cell parts $(44,364)$, and organelles $(31,422)$. Most transcripts enriched in molecular processes (MF) were binding $(49,208)$, catalytic activity $(29,970)$, and transporter activity $(4,766)$. For biological processes (BP), transcripts were mainly enriched in cellular processes $(45,934)$, single-organism processes $(37,336)$, and metabolic processes $(33,199)$.

In this study, 99,079 transcripts were annotated by blasting with linear homologous groups in the eggNOG database for function description and classification. The annotation results were classified into 25 categories (Figure 2C). The three largest groups were post-translational modification, protein turnover, chaperones $(10,389)$, intracellular trafficking, secretion, vesicular transport $(9,355)$, and signal transduction mechanisms $(6,657)$ followed by transcription $(6,166)$, cytoskeleton $(3,078)$, and translation, ribosomal structure, and biogenesis $(2,545)$.

Based on the KEGG analysis, 67,965 transcripts were enriched in 297 pathways. The five pathways enriched with the most genes were endocytosis $(2,214)$, protein processing in endoplasmic reticulum $(1,658)$, regulation of actin cytoskeleton $(1,641)$, herpes simplex infection $(1,533)$, and MAPK signaling pathway $(1,518)$ (Figure 2D).

\section{Differentially Expressed Genes (DEGs) Analysis}

The expression levels of genes in five tissues of immature and mature lenok were investigated, including the gonad (G), head kidney $(\mathrm{K})$, liver $(\mathrm{L})$, muscle $(\mathrm{M})$, and spleen $(\mathrm{S})$. The numbers of upregulated and downregulated DEGs between the immature and mature groups are shown in Table 3. 
A

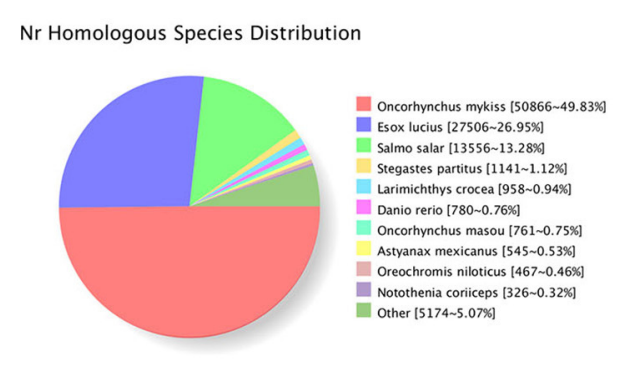

B

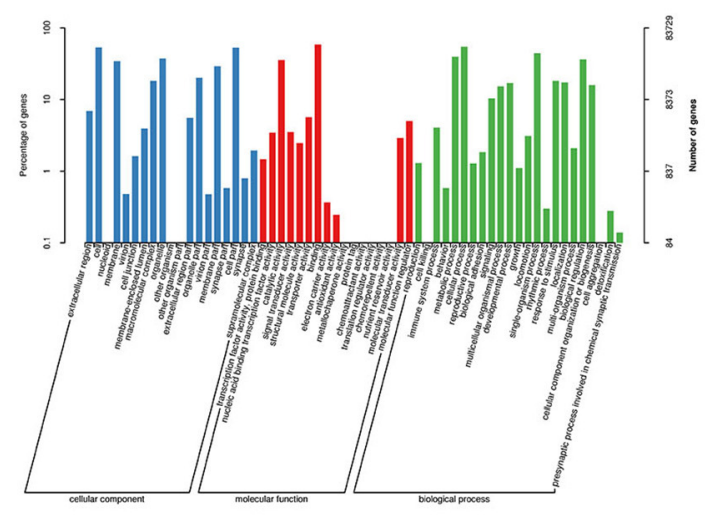

C

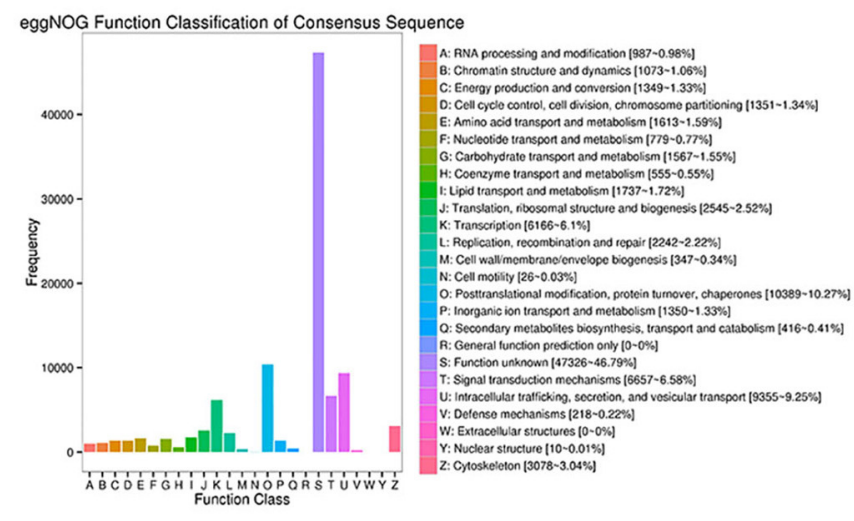

D

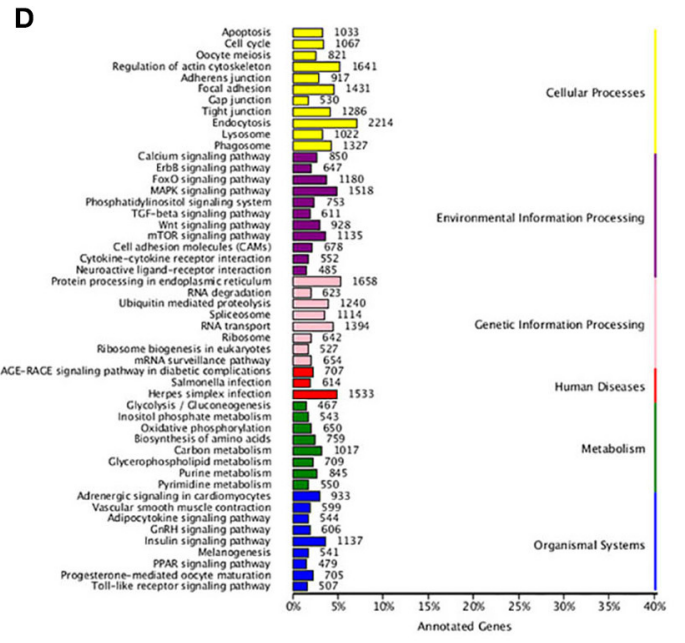

FIGURE 2 | Functional annotation information of non-redundant transcripts. (A) Homologous species distribution of transcripts in NR database; (B) GO analysis of annotated transcripts; (C) eggNOG function classification of transcripts; (D) KEGG pathway classified analysis of transcripts.

TABLE 3 | Statistics for DEGs of five tissues between of immature and mature Brachymystax lenok.

\begin{tabular}{llll}
\hline DEG Set (immature vs. mature) & All DEGs & Up-regulated & Down-regulated \\
\hline Gonad vs. M gonad & 7,728 & 3,306 & 4,422 \\
Head kidney vs. M head kidney & 10,352 & 3,840 & 6,512 \\
Liver vs. M liver & 6,690 & 2,254 & 4,436 \\
Muscle vs. M muscle & 7,968 & 3,268 & 4,700 \\
Spleen vs. M spleen & 10,837 & 4,089 & 6,748 \\
\hline
\end{tabular}

We compared the DEGs between the immature and mature groups in the five tissues. Compared with the mature group, 719 genes with significantly increased expression (Figure 3A) and 1,727 genes with significantly decreased expression (Figure 3B) in all five tissues were found in the immature group. To investigate the function of DEGs, a KEGG analysis was performed on the overlapping genes. The results showed that 291 of 719 upregulated overlapping DEGs were enriched in the KEGG pathway, among which the significantly enriched pathways were carbon metabolism (23), pyruvate metabolism (11), and protein processing in the endoplasmic reticulum (30) (Figure 3C). Among the 1,727 downregulated overlapping DEGs, 693 genes were enriched in the KEGG pathway, among which the enriched pathways included spliceosome (53) and protein processing in the endoplasmic reticulum (65) (Figure 3D).

\section{Pathways Related to Endocrine System and Development}

The endocrine system and development-related pathways play important roles in the process of gonadal maturation (38). According to the classification information of the KEGG database (https://www.kegg.jp/kegg/pathway.html), the second classifications of endocrine system and development were under the primary classification of organismal systems. We analyzed the enriched DEGs in the pathways related to the endocrine system and development and their expression patterns in different tissues of immature and mature groups. A total of 77 DEGs were screened for the enrichment of these two classifications, and they enriched the adipocytokine signaling 


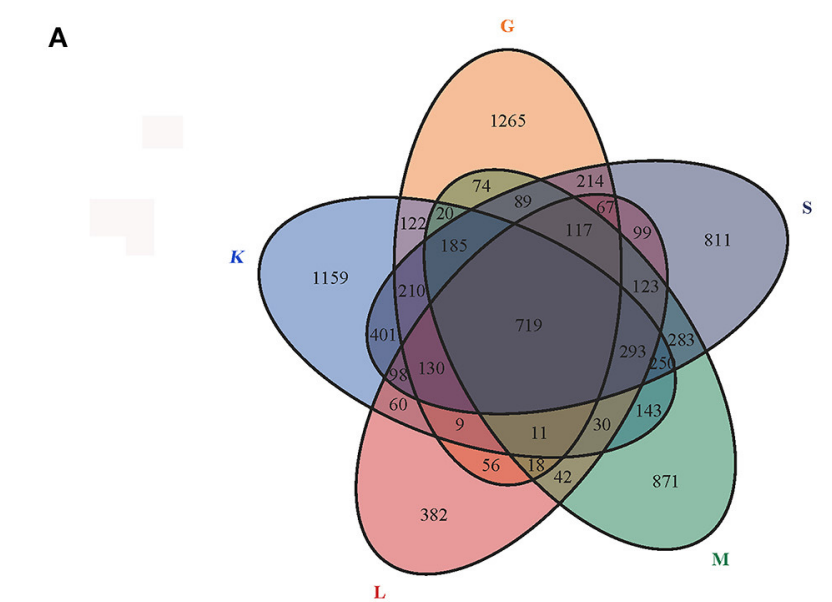

C

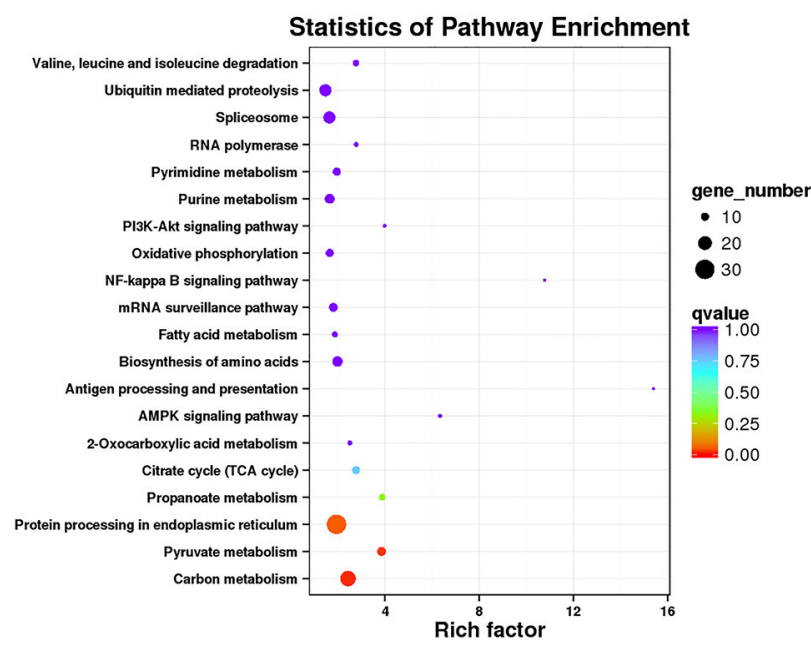

B

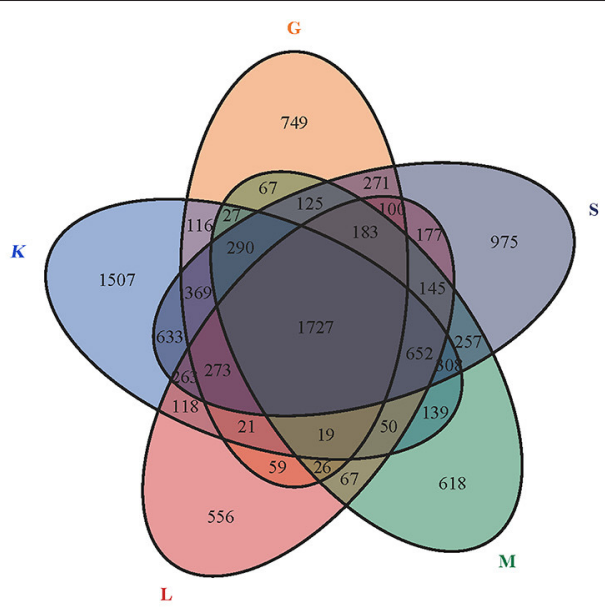

D

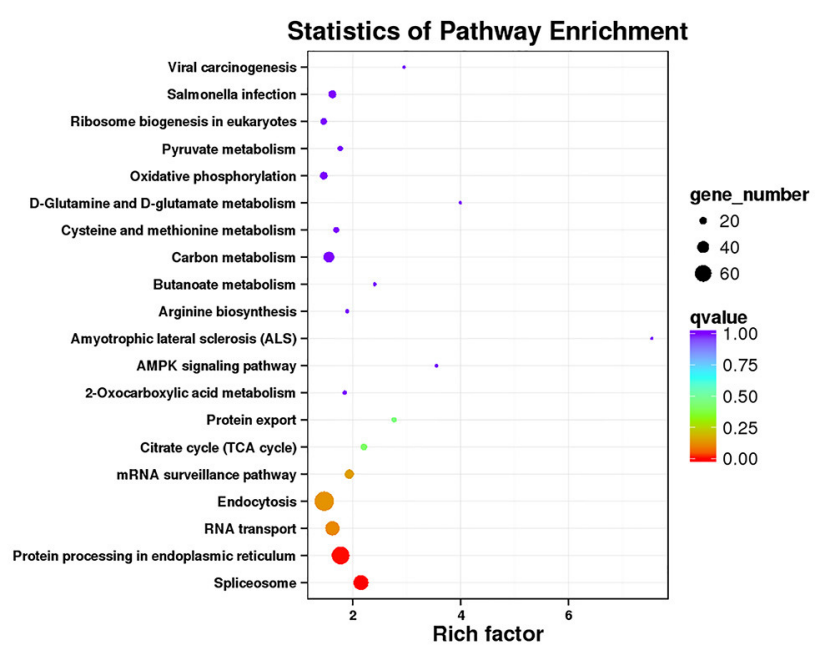

FIGURE 3 | Expression analysis of DEGs in five tissues of lenok. (A) Upregulated DEGs; (B) Downregulated DEGs; K, head kidney; L, liver; M, muscle; S, spleen; G, gonad; (C) KEGG analysis of upregulated DEGs; (D) KEGG analysis of downregulated DEGs.

pathway (ko04920), GnRH signaling pathway (ko04912), insulin signaling pathway (ko04910), PPAR signaling pathway (ko03320), progesterone-mediated oocyte maturation (ko04914), and renin secretion (ko04924). Information regarding the DEGs enriched in these pathways is presented in Supplementary Table S2, and their expression profiles in different tissues are shown in Figure 4. There were 31 DEGs enriched in the insulin signaling pathway, followed by the adipocytokine signaling pathway [16], PPAR signaling pathway [12], GnRH signaling pathway [10], progesterone-mediated oocyte maturation [9], renin secretion [2], and only one DEG was enriched in the remaining pathways.

Among the 77 DEGs enriched in the above pathways, the expression of 69 DEGs increased in mature lenok, indicating that the expression of genes involved in development and the endocrine system increased with the growth and development of lenok. Among the DEGs enriched in the above pathways, the five genes that expressed the most significant differences in the gonads were F01_transcript_1427 (unknown function), F02_transcript_5540 (p38 mitogen-activated protein kinase), F01_transcript_61649 (unknown function), F01_transcript_83061 (guanine nucleotide-binding protein subunit alpha-11), and F01_transcript_10752 (5-AMP-activated protein kinase) (Table 4).

\section{Verification of RNA-seq by qRT-PCR}

To verify the accuracy of RNA-seq, 12 DEGs were randomly selected for qRT-PCR verification, and their primer information is presented in Supplementary Table S3. The results of RNA-seq and qRT-PCR of these 12 DEGs in the five tissues are shown in Figure 5, and the correlation was expressed by Pearson's coefficient $\left(r^{2}=0.9521\right)$. The results showed consistency and correlation between the results of RNA-seq and qRT-PCR, which proved the effectiveness of RNA-seq. 


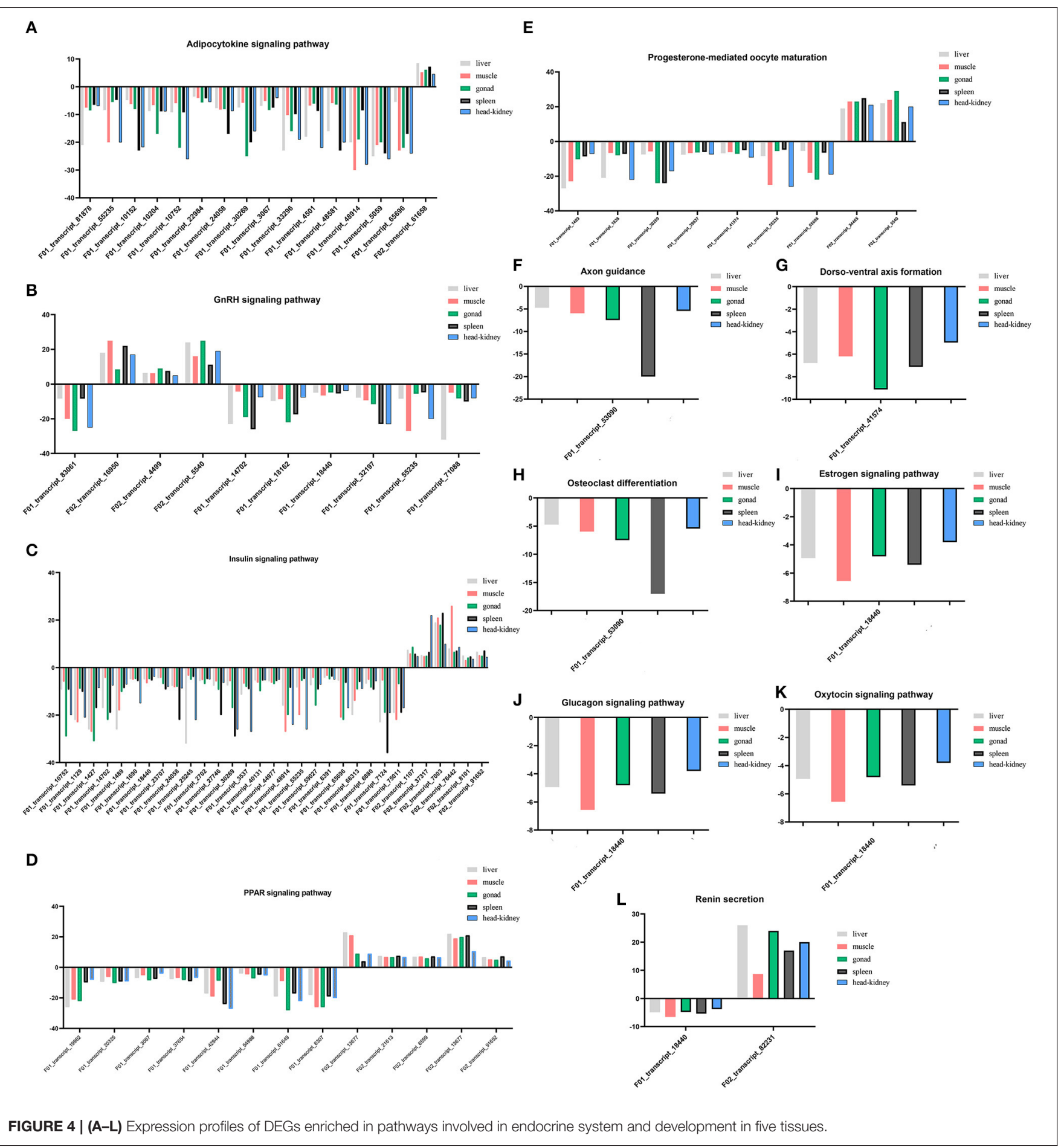

\section{Validation Analysis}

\section{Expression Analysis of p38 MAPK in B. lenok}

Transcriptome sequencing results showed that p38 MAPK was significantly differentially expressed in mature and immature gonads (Table 4). To study the expression pattern of p38 MAPK in lenok, the expression of p38 MAPK protein was detected in eight tissues of lenok, including the gill, heart, liver, spleen, intestine, brain, skin, and gonads (Figure 6A), and the results showed that the expression of p38 MAPK was higher in the intestine, heart, and brain tissues, but was not expressed in the gill and spleen. The expression levels of p38 MAPK protein were also detected in the ovaries of lenok at different developmental stages. The results showed that the expression of p38 MAPK at $750 \mathrm{dph}$ was 2.8 -fold that at $300 \mathrm{dph}$, and the expression at three 
TABLE 4 | Specifically expressed genes of the top five |log2FC| in gonad.

\begin{tabular}{|c|c|c|}
\hline Gene & $|\log 2 F C|$ & Description \\
\hline F01_transcript_1427 & -31.50 & Protein of unknown function \\
\hline F02_transcript_5540 & -29.78 & p38 mitogen activated protein kinase \\
\hline F01_transcript_61649 & -28.96 & Protein of unknown function \\
\hline F01_transcript_83061 & -26.47 & Guanine nucleotide-binding protein subunit alpha-11 \\
\hline F01_transcript_10752 & 29.37 & 5'-AMP-activated protein kinase \\
\hline
\end{tabular}

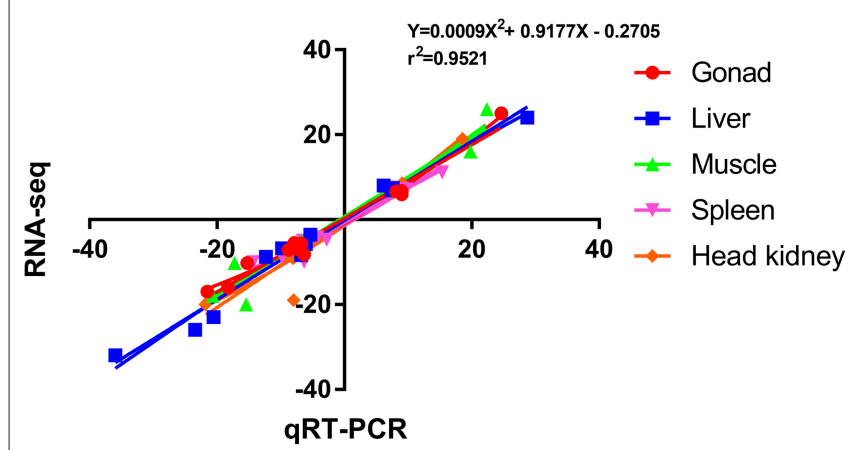

FIGURE 5 | Scatterplot of the correlation between qRT-PCR and RNA-seq. $X$-axis, qRT-PCR; Y-axis, RNA-seq; r2, Pearson correlation coefficient.

years ph was 2.0-fold of that at $750 \mathrm{dph}$ and 3.1-fold of that at 300 $\mathrm{dph}$ (Figure 6B). The results showed that the expression of $\mathrm{p} 38$ MAPK in the ovary significantly increased with the growth and development of lenok.

\section{Effects of p38 MAPK on Ovarian Development of B. lenok}

To verify the regulatory effect of p38 MAPK on ovarian development in lenok, an inhibitor of SB203580 was injected intraperitoneally to inhibit $\mathrm{p} 38 \mathrm{MAPK}$ protein phosphorylation. The results showed that the expression levels of p38 MAPK protein in the SB203580 inhibitor, DMSO, and control groups were not significantly different (Figure 7A). However, the phosphorylation levels of p38 in the SB203580 inhibitor group were 0.32 -fold that of the control group and 0.55 times that of the DMSO group, indicating a significant decreasing trend (Figure 7B).

To explore the effect of the p38 MAPK pathway on the balance of hormones in the hypothalamic-pituitary-gonadal (HPG) axis of lenok, gonadotropin-releasing hormone 3 (GnRH3), follicle stimulating hormone (FSH), luteinizing hormone ( $\mathrm{LH})$, and oestradiol (E2) hormone levels were detected in the plasma of lenok in the SB203580 inhibitor, DMSO, and control groups, respectively. The values of OD450 in different groups are shown in Supplementary Table S4. The repetition rate of the GnRH3 assay was $1.7-7.8 \%$, the FSH was $1.9-9.6 \%$, the LH was $1.3-4.7 \%$, and the E2 was $1.8-7.8 \%$. The variable coefficients of all samples were no greater than $10 \%$, proving that our ELISA results had good repeatability.
The results showed that the level of GnRH3 in the inhibitor group was 1.4-fold higher than that in the control group ( $P$ $<0.05)$ and 1.9-fold higher than that in the DMSO group $(P$ $<0.05$ ), and there was no significant difference between the control and DMSO groups $(P>0.05)$. The FSH level in the inhibitor group was 0.6 -fold lower than that in the control group $(P<0.05)$, and there was no significant difference between the inhibitor and DMSO groups $(P>0.05)$. The LH levels in the three groups were not significantly different $(P>0.05)$. The level of E2 in the inhibitor group was significantly lower than that in the control and DMSO groups, which was 0.4 -fold higher than that in the control group $(P<0.01)$ and 0.5 -fold higher than that in the DMSO group $(P<0.05)$ (Figure 7C).

Immunohistochemical sections labeled with p38 MAPK protein were used to explore the morphological changes in lenok ovaries after p38 MAPK activity was inhibited. There were oocytes at phases II and III in the control group. The cell volume was larger, and the cytoplasm increased with the nucleus inside. A flat layer of nucleolar structure overlapped the oocyte and the theca cells around the follicle were complete (Figure 7D). In the DMSO group, the morphology of follicles was almost consistent with that of the control group, and only theca cells appeared irregular (Figure 7E), which may be because of the toxic effect of DMSO on the ovary. In the inhibitor group, the structure of follicles was obviously changed, with the rupture of cell nucleus, vacuoles in follicles, and atrophy of theca cells, which showed irregular arrangement (Figure 7F).

\section{DISCUSSION}

In this study, SMRT technology based on the PacBio platform was used to sequence the mixed tissue samples of immature and mature lenok, including the liver, muscle, head kidney, gonad, and spleen. SMRT technology is superior to next-generation sequencing technologies such as Illumina, which can conduct de novo sequencing of the complete mRNA to obtain the full-length information of the transcripts directly. This method can provide accurate reference sequences, overcome the problems of short transcription splicing and incomplete information of species without reference genomes, and contribute to the screening and identification of functional genes. As a rare cold-water fish native to China, the wild population of $B$. lenok is decreasing annually. The analysis of genetic mechanisms plays an important role in the establishment of artificial breeding technology and genetic improvement of lenok. As a non-model organism, the identification of functional genes in lenok is limited because of 
A
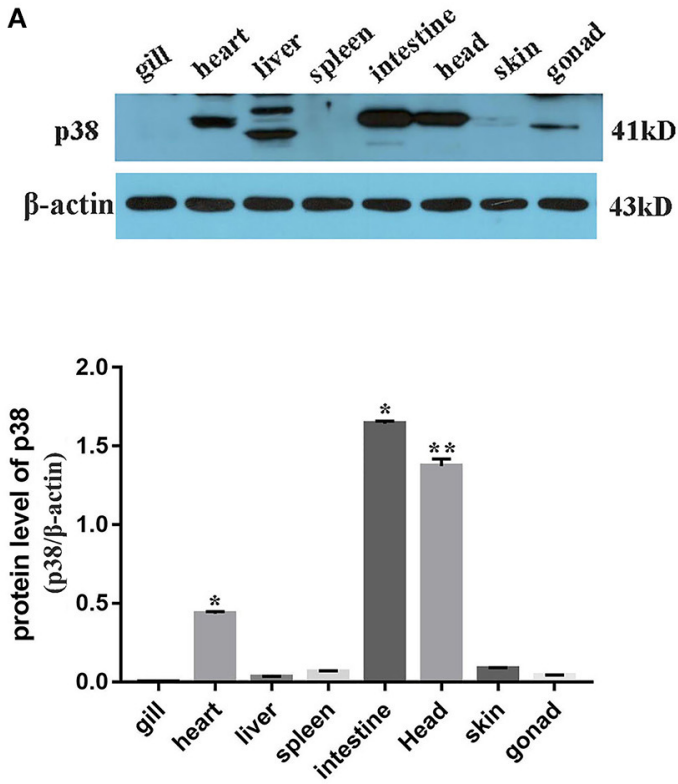

B
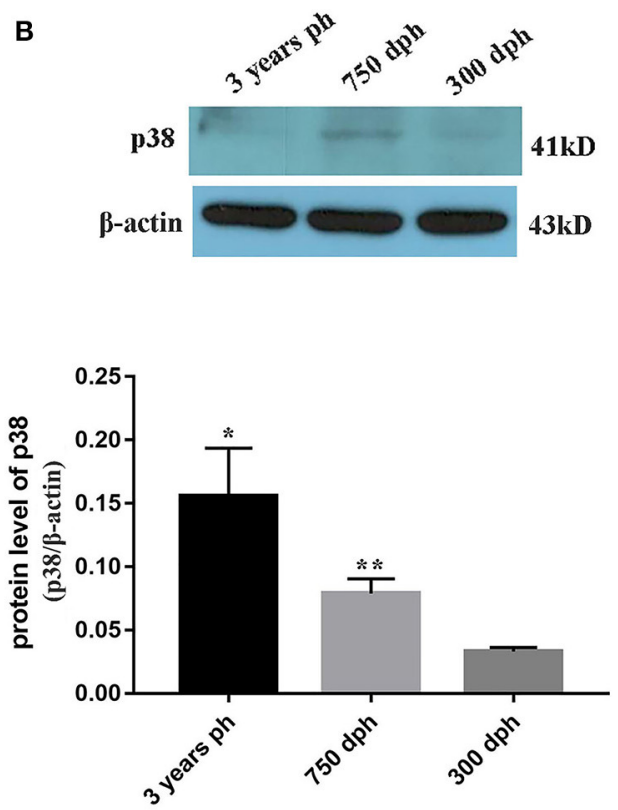

FIGURE 6 | Expression pattern of p38 MAPK protein in B. lenok. (A) The expression level of p38 MAPK protein in 8 tissues. The significance was expressed "*” as a $P$-value of $<0.05$ vs. gill, and "*ᄎ" as a $P$-value of $<0.01$ vs. gill. (B) The expression level of p38 MAPK protein in gonad at different developmental stages. The

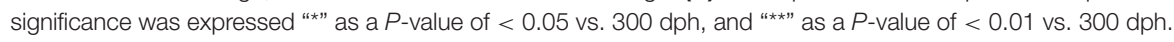

the lack of a reference genome. In this study, key genes and pathways related to the gonadal development of lenok were screened using SMRT sequencing and corrected using Illumina sequencing, and the function of the gene involved in ovarian development of lenok was explored.

The Iso-seq results showed that the lengths of the circular consensus sequence (CCS) reads were 2,791 and 2,977, respectively, and the percentage of FLNC reads with the $5^{\prime}$ end, $3^{\prime}$ end, and poly-A structures were 84.09 and $86.36 \%$, respectively, for mature and immature samples. The length of N50 and proportion of FLNC were better than those of red swamp crayfish (39) and Hong Kong catfish (40). After NGS correction, 61,405 and 59,372 non-redundant transcripts were obtained for the mature and immature groups, respectively, for subsequent expression levels and pathway enrichment analysis. These transcripts were evaluated by BUSCO, and the transcripts that encoded complete proteins accounted for 85.69 and $83.68 \%$ in the mature and immature groups, respectively. The proportion of complete transcripts in this study was higher than that obtained from the full-length transcriptome of other aquatic animals, such as Atlantic bluefin tuna (80\%) (41) and shrimp (81\%) (11). These results proved that our sequencing results were of high quality and were reliable for the analysis of functional gene information.

Eukaryotic transcription factors can specifically bind to the upstream sequence of the 5 '-end of a specific gene, thus ensuring that the gene is expressed at a specific intensity at a specific time and space (42). Studies have shown that TFs play important roles in fish morphogenesis (43), growth and development (44), gonadal maturation (45), and immune regulation (46).
The sequencing results of the full-length transcriptome in this study showed that the TFs of the zf-C2H2, ZBTB, BHLH, homeobox, miscellaneous, TF-BZIP, and HMG families appeared in large numbers, suggesting that they play pivotal roles in the growth and development of lenok. The $\mathrm{C} 2 \mathrm{H} 2$ zinc finger (zf$\mathrm{C} 2 \mathrm{H} 2$ ) proteins are the most abundant transcriptional regulatory factors in mammals. Most of the zinc finger motifs of $\mathrm{zf}-\mathrm{C} 2 \mathrm{H} 2$ proteins are not conserved, indicating that they may bind to different DNA sequences to regulate different genes and perform diverse regulatory functions. Most human $\mathrm{zf}-\mathrm{C} 2 \mathrm{H} 2$ proteins are completely different from those of other species, such as mice, so the function of $\mathrm{zf}-\mathrm{C} 2 \mathrm{H} 2$ proteins is not consistent between different species (47). In this study, we concluded that the zf$\mathrm{C} 2 \mathrm{H} 2$ protein was the most abundant type of TF in lenok, and its specific function should be further studied. The ZBTB family refers to a class of proteins containing the $\mathrm{N}$-terminal $\mathrm{BTB}$ domain and multiple zinc finger domains at the C-terminal (48), and more than 60 types of ZBTB proteins have been identified as being involved in development, differentiation, and tumor formation (49). Recent studies have shown that $z b t b 16$ can regulate spermatogenesis by controlling the self-renewal and repair of spermatogonia $(50,51)$. In orange-spotted grouper, $z b t b 40$ is specifically expressed in male germ cells and regulates spermatogenesis through its interaction with cyp17a1 (49).

Different tissues play important regulatory roles in the growth and development of fish. Through transcriptome screening, DEGs were identified in the gonad, head kidney, liver, muscle, and spleen tissues between mature and immature lenok groups. Most DEGs were found in the spleen $(10,837)$, followed by the head kidney $(10,352)$, muscle $(7,968)$, gonad $(7,728)$, 
A
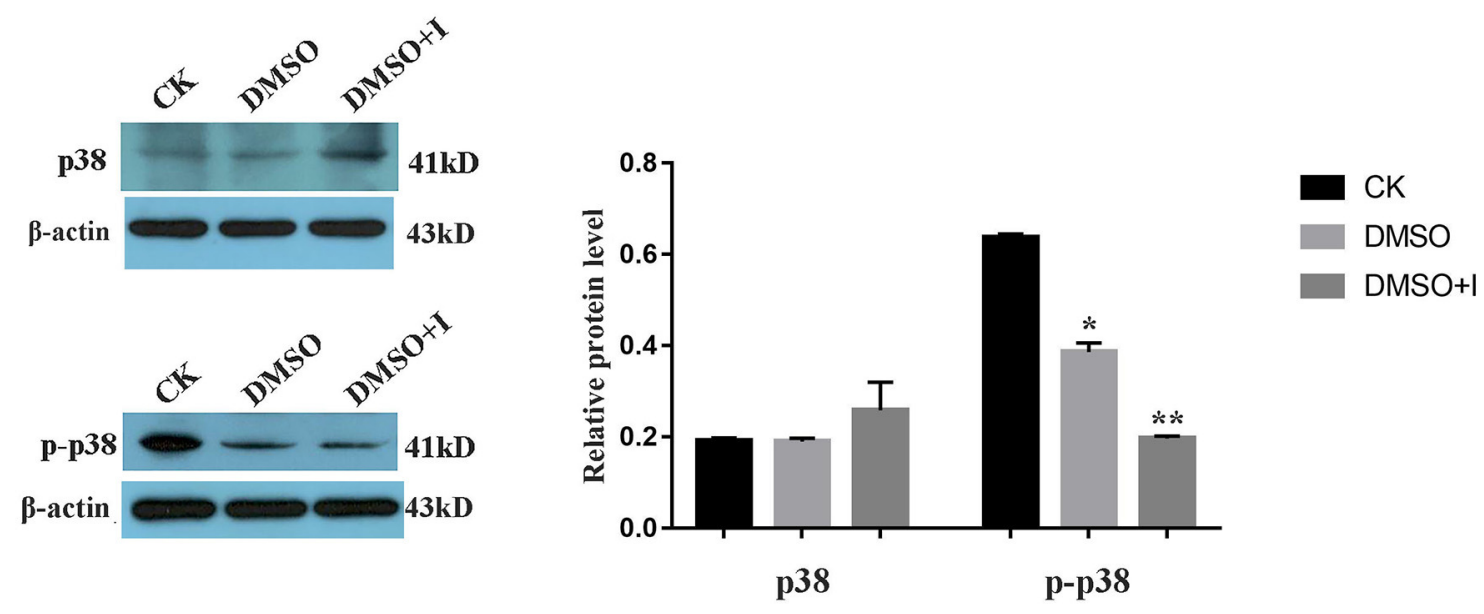

C

GnRH3

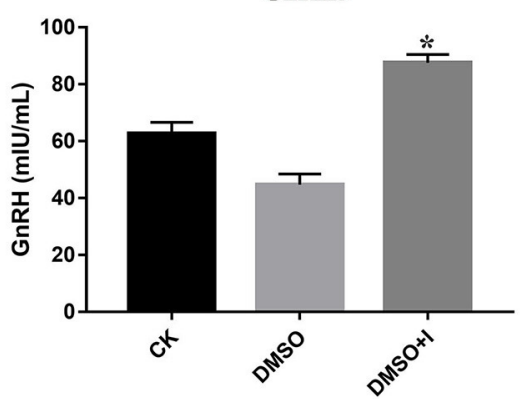

- $\mathrm{CK}$

DMSO

DMSO+1

LH

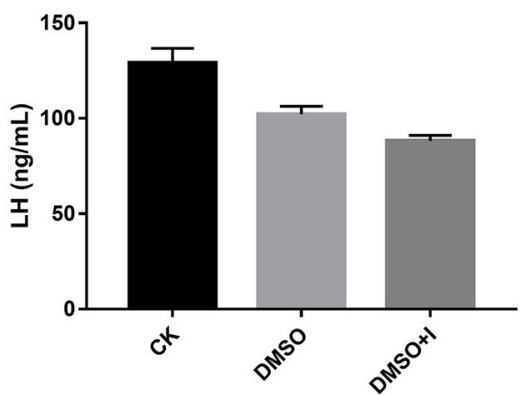

- $\mathrm{CK}$

DMSO

DMSO+I
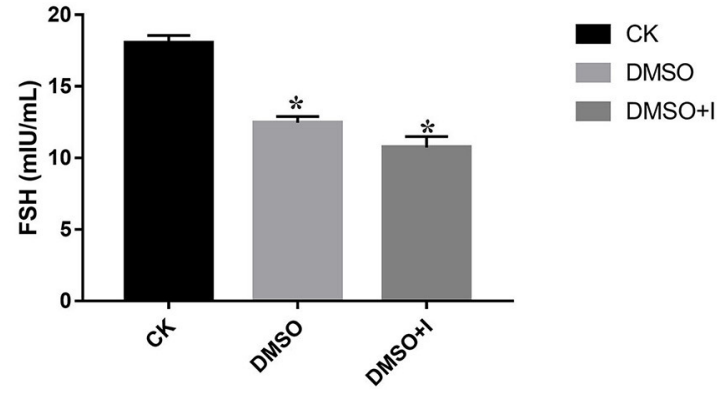

D

CK

E
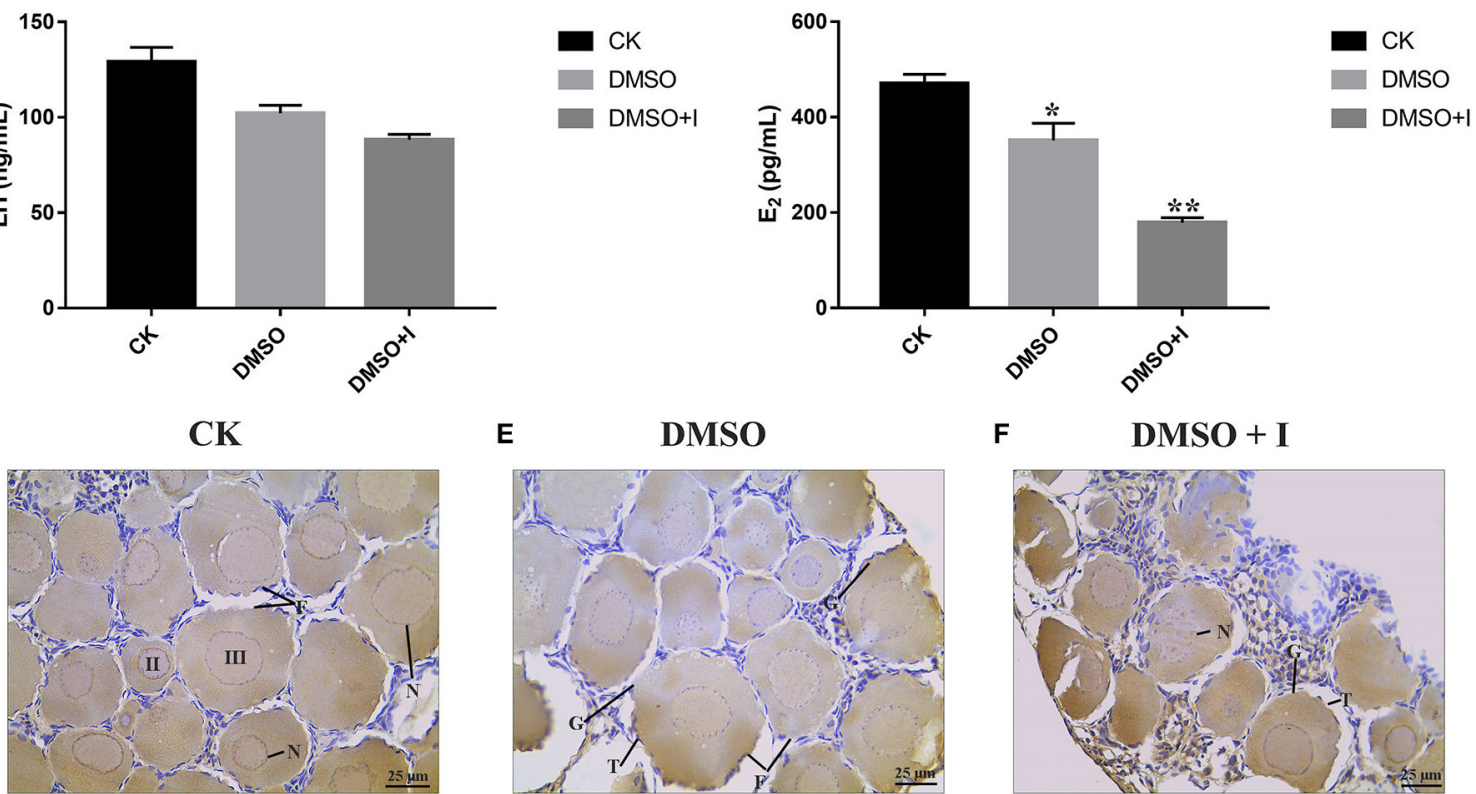

$\mathbf{F}$

DMSO + I

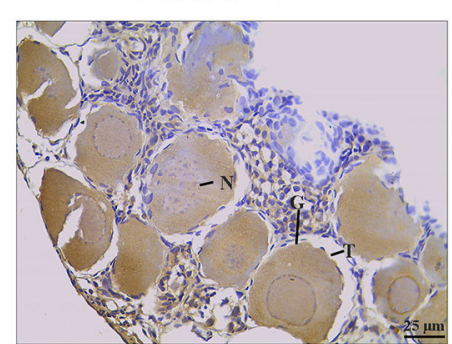

FIGURE 7 | Effects of p38 MAPK on ovarian development of B. lenok. (A) The expression level of p38 MAPK protein in ovary; (B) The phosphorylation level of p38 MAPK protein in ovary of different groups; (C) The level of HPG hormones of different groups; The significance was expressed "*” as a $P$-value of $<0.05$ vs. CK

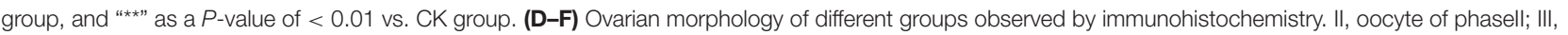
oocyte of phase III; F, follicle; T, theca cell; G, granulosa cell; N, nucleus. 
and liver $(6,690)$. The spleen and head kidney are important haematopoietic tissues in fish and play important roles in the generation, storage, and maturation of red blood cells and granulocytes $(52,53)$. The head kidney also contains phagocytes and $\mathrm{B}$ cells, and is an important organ for the production of antibodies (54). In many fish, the ability of specific immunity gradually increases with growth and development (55), and therefore, the number of DEGs in the spleen and head kidney was higher. Compared with the mature group, the number of downregulated DEGs was higher than that of the upregulated DEGs in the immature group, which indicated that the expression of most genes showed a significantly increased trend with the growth and development of lenok.

Due to the rapid decline in natural resources, it is of great practical significance to conduct the artificial reproduction and genetic breeding of lenok. Therefore, it is of great importance to understand the regulatory mechanisms of gonadal development and maturation of lenok. Gonadal developmental processes, such as germline generation, proliferation, and yolk accumulation in fish are regulated by the endocrine system (56). DEGs enriched in pathways involved in the endocrine system were analyzed, and most DEGs were found to be enriched in the insulin signaling pathway (ko04910) and the peroxisome proliferatoractivated receptor (PPAR) signaling pathway (ko03320). Insulin plays an important role in the reproductive process of female animals and has direct and indirect effects on the production of ovarian steroid hormones and the growth of granulosa and theca cells (57). There are many important effectors in the insulin pathway, including insulin receptor (IR), insulin receptor substrate (IRS), phosphatidylinositol 3 kinase (PI3K), and protein kinase B (AKT). IR expression has been detected in granulosa and theca cells and follicles in a variety of animals (58-60) at different stages of development (61). PI3K can regulate Akt to participate in many physiological processes, and the PI3K/Akt pathway plays key regulatory roles in follicular structural differentiation, growth, and development (62). In this study, we preliminarily showed that the insulin signaling pathway plays an essential role in the regulation of the ovarian development in lenok. PPARs are important in the reproductive system, particularly in the HPG axis (63). Some studies have demonstrated that PPARs can regulate ovum proliferation, tissue remodeling, and hormone synthesis (64).

The expression patterns of DEGs enriched in endocrine and development-related pathways were analyzed in the gonads of lenok. The results indicated that p38 MAPK, GNA11, and AMPK were significantly differentially expressed between mature and immature gonads, but the functions of these genes in lenok remain unknown. As a central substance of cell energy metabolism, adenylate activated protein kinase (AMPK) plays a non-negligible role in the process of ovarian development (65). Glucose and adiponectin promote AMPK phosphorylation (66), whereas IGF-1 and FSH inhibit AMPK phosphorylation (67). Phosphorylated AMPK promotes oocyte development, but negatively regulates follicle and granulosa cell development (68). As one of the main branches of the MAPK signaling pathway, p38 MAPK can be activated in response to a variety of environmental stresses or inflammatory stimuli, thereby promoting apoptosis and inhibiting cell growth (22). However, research on lenok remains in its infancy. In this study, the expression of p38 MAPK protein was detected in different tissues of lenok, and the results showed that p38 MAPK plays a role in the regulation of intestinal, heart, and brain tissues. The expression pattern of p38 MAPK protein was simultaneously explored at different developmental stages of the lenok ovary. Under the same artificial feeding conditions, the expression levels of p38 MAPK protein increased gradually with the growth and development of lenok.

SB203580 is a pyridine imidazole derivative that can inhibit the catalytic activity of p38 MAPK by binding competitively to the ATP sites (69), and thus, can specifically inhibit the p38 MAPK signaling pathway $(70,71)$. The inhibitor was injected intraperitoneally to investigate the effects of p38 MAPK on steroid hormone levels and follicular structures of lenok. The results showed that under the influence of the inhibitor SB203580, the phosphorylation levels of p38 MAPK in the ovary were significantly decreased, indicating that SB203580 blocked the p38 MAPK signaling pathway. We also investigated the influence of p38 MAPK pathway inhibition on the HPG axis of lenok. The synthesis and secretion of GnRH in the hypothalamus promotes LH and FSH synthesis and secretion in the pituitary, thereby stimulating the synthesis and secretion of E2 in the ovary (72). Hormones in the HPG axis maintain normal development of the ovary and oogenesis and regulate the physiological functions of the reproductive system of fish (73). The levels of major reproductive hormones in the plasma were monitored to explore the effects of p38 MAPK signaling pathway inhibition on lenok reproductive hormone balance. Oocytes at $300 \mathrm{dph}$ (immature) ovaries were in the early stage, while a large amount of yolk existed in follicles at three years ph (mature), which would have a substantial influence on histological experiments. However, oocytes of various stages existed in the ovary of lenok at $750 \mathrm{dph}$, which was more suitable for function verification.

$\mathrm{GnRH}$ is synthesized and secreted by GnRH neurons in the medial hypothalamus and maintains hormone balance and homeostasis through autocrine and paracrine mechanisms under the synergistic effect of related hormones (74-76). Compared with the CK and DMSO groups, the GnRH3 level in the inhibitor group increased significantly, indicating that the hormone level of the HPG axis was abnormal, resulting in the continuous secretion of GnRH3 hormone in the hypothalamus. However, no serious tissue damage was caused to the hypothalamus. FSH and $\mathrm{LH}$ are secreted by the anterior pituitary gland, and FSH binds to specific receptors and stimulates the formation of $\mathrm{LH}$ receptors $(77,78)$. In addition, FSH acts synergistically with LH to promote follicle maturation and stimulate E2 synthesis and secretion in the ovary $(79,80)$. In this study, it was found that compared with the CK group, FSH levels were significantly decreased in the DMSO group, which may be caused by toxicity of DMSO. In the inhibitor group, FSH levels were significantly decreased, whereas there was no significant effect on LH levels. Previous studies have shown that inhibition of the p38 MAPK pathway can weaken the stimulatory effect of FSH on E2 and cytochrome P450 aromatase (81). Combined with the results of this study, it was confirmed that the p38 MAPK pathway is involved in the synthesis and function of FSH in lenok. 
E2 is a steroid hormone produced by follicular theca cells and is the main estrogen of teleosts (82). It plays important reproductive roles, such as promoting follicular development and regulating the oestrus cycle (83). The results of this experiment showed that the level of E2 in the inhibitor group was significantly lower than that in the CK group. Previous studies have shown that inhibition of p38 MAPK activity can inhibit the generation of E2 (84). In addition, combined with the results of immunohistochemistry in this study, inhibition of p38 MAPK activity could cause atrophy and irregular arrangement of granulosa and theca cells, where E2 was mainly secreted. Thus, inhibition of p38 MAPK activity could result in morphological and functional changes in the granulosa and theca cells of lenok and affect the synthesis and secretion of E2, leading to HPG axis blocking and abnormal follicular development. Therefore, the p38 MAPK pathway plays an important role in maintaining the balance between reproductive hormones and follicle development in lenok.

\section{CONCLUSION}

This is the first comparative transcriptome analysis of $B$. lenok combined with SMRT and NGS, and for the first time, the DEGs between immature and mature lenok were analyzed in five tissues. Furthermore, DEGs and pathways involved in the endocrine system and gonadal development were identified, and p38 MAPK was identified to potentially regulate gonadal development in lenok. Inhibiting the activity of p38 MAPK blocked the HPG axis and abnormal follicular development in lenok. Our study illustrates the basic regulatory mechanism of ovarian development and provides a reference for genetic improvement in lenok.

\section{DATA AVAILABILITY STATEMENT}

The datasets presented in this study can be found in online repositories. The names of the repository/repositories and accession number(s) can be found below: https://www. ncbi.nlm.nih.gov/, PRJNA669274 and https://www.ncbi.nlm.nih. gov/, PRJNA669219.

\section{REFERENCES}

1. Wang S, Zheng GM, Wang QS. China Red Data Book of Endangered Animals: Pisces (in Chinese with English Translation). Science Press, Beijing. (1998).

2. Chang J, Niu HX, Jia YD, Li SG, Xu GF. Effects of dietary lipid levels on growth, feed utilization, digestive tract enzyme activity and lipid deposition of juvenile Manchurian trout, Brachymystax lenok (Pallas). Aquaculture Nutrition. (2017) 24:694-701. doi: 10.1111/anu.12598

3. Yu J, Li S, Chang J, Niu H, Hu Z, Han Y. Effect of variation in the dietary ratio of linseed oil to fish oil on growth, body composition, tissues fatty acid composition, flesh nutritional value and immune indices in Manchurian trout, Brachymystax lenok. Aquaculture Nutr. (2019) 25:37787. doi: $10.1111 /$ anu. 12863

4. Olson KW, Jensen OP, Hrabik TR. Feeding ecology and prey resource partitioning of lenok (B rachymystax lenok) and Baikal grayling ( $\mathrm{T}$ hymallus

\section{ETHICS STATEMENT}

The animal study was reviewed and approved by all experiments were performed according to the European Communities Council Directive (86/609/EEC). The performances were approved by the Animal Husbandry Department of Heilongjiang Animal Care and Use Committee (202110384464).

\section{AUTHOR CONTRIBUTIONS}

TH designed and performed the experiments. GX analyzed the data and checked the manuscript. WG, LZ, WJ, and CL cultured and sampled the fish. EL and FD drafted the manuscript. XH and BW reviewed the manuscript. All authors contributed to the article and approved the submitted version.

\section{FUNDING}

This study was supported by the China Agriculture Research System of MOF and MARA (CARS-46) and the Central Publicinterest Scientific Institution Basal Research Fund, CAFS (No. 2020TD32). The funding agency played no part in the study design, data collection and analysis, decision to publish, or manuscript preparation.

\section{SUPPLEMENTARY MATERIAL}

The Supplementary Material for this article can be found online at: https://www.frontiersin.org/articles/10.3389/fvets. 2022.752521/full\#supplementary-material

Supplementary Figure S1 | The correlation of samples for next generation sequencing.

Supplementary Figure S2 | The standard curves of Elisa kits for GnRH3, LH, $\mathrm{FSH}$, and $\mathrm{E} 2$ in lenok.

Supplementary Table S1 | The analysis of SSR by MISA.

Supplementary Table S2 | The information about the DEGs enriched in pathways involved in endocrine systems and developments.

Supplementary Table S3 | The primer information of DEGs selected for qRT-PCR verification.

Supplementary Table S4 | The results of intra-assay repeat tests of ELISA. arcticus baicalensis) in the Eg and Uur rivers. Mongolia. (2016) 25:565576. doi: 10.1111/eff.12234

5. Tomas S, Underwood JG, Tseng E, Holloway AK. Long-read sequencing of chicken transcripts and identifcation of new transcript isoforms. PLoS ONE. (2014) 9:e94650. doi: 10.1371/journal.pone.00 94650

6. Abdel-Ghany SE, Hamilton M, Jacobi JL, Ngam P, Devitt N, Schilkey F, et al. A survey of the sorghum transcriptome using single-molecule long reads. Nat Commun. (2016) 7:11706. doi: 10.1038/ncomms11706

7. Wang B, Tseng E, Regulski M, Clark TA, Hon T, Jiao Y, et al. Unveiling the complexity of the maize transcriptome by single-molecule long-read sequencing. Nat Commun. (2016) 7:11708. doi: 10.1038/ncomms11708

8. Larsen PA, Smith TP. Application of circular consensus sequencing and network analysis to characterize the bovine Ig G repertoire. BMC Immunol. (2012) 13:52. doi: 10.1186/1471-2172-13-52 
9. Chen SY, Deng F, Jia X, Li C, Lai SJ. A transcriptome atlas of rabbit revealed by PacBio single-molecule long-read sequencing. Sci Reports. (2017) 7:7648. doi: 10.1038/s41598-017-08138-z

10. Treutlein B, Gokce O, Quake SR, Südhof TC. Cartography of neurexin alternative splicing mapped by single-molecule long-read mRNA sequencing. Proc Natl A-cad Sci USA. (2014) 111:1291-9. doi: 10.1073/pnas.1403244111

11. Zeng D, Chen X, Peng J, Yang C, Peng M, Zhu W, et al. Single-molecule longread sequencing facilitates shrimp transcriptome research. Sci Reports. (2018) 8:16920. doi: 10.1038/s41598-018-35066-3

12. Nudelman G, Frasca A, Kent B, Sadler KC, Sealfon SC, Walsh MJ, et al. High resolution annotation of zebrafish transcriptome using longread sequencing. Genome Res. (2018) 28:1415-25. doi: 10.1101/gr.223586.117

13. Feng X, Jia Y, Zhu R, Chen K, Chen Y. Characterization and analysis of the transcriptome in Gymnocypris selincuoensis on the Qinghai-Tibetan Plateau using single-molecule long-read sequencing and RNA-seq. DNA Res. (2019) 26:353-63. doi: 10.1093/dnares/dsz014

14. Tian Y, Wen H, Qi X, Zhang X, Liu S, Li B, et al. Characterization of fulllength transcriptome sequences and splice variants of Lateolabrax maculatus by singlemolecule long-read sequencing and their involvement in salinity regulation. Front Genet. (2019) 10:1126-45. doi: 10.3389/fgene.2019.01126

15. Yang HX, Zhou Y, Gu JL, Xie SY, Xu Y, Zhu GF, et al. Deep mRNA sequencing analysis to capture the transcriptomelandscape of zebrafish embryos and larvae. PLoS ONE. (2013) 8:64058. doi: 10.1371/journal.pone.0064058

16. Sørhus E, Incardona JP, Furmanek T, Jentoft S, Meier S, Edvardsen RB. Developmental transcriptomics in Atlantic haddock: Illuminating pattern formation and organogenesis in non-model vertebrates. Dev Biol. (2016) 411:301-13. doi: 10.1016/j.ydbio.2016.02.012

17. Zeng QF, Liu SK, Yao J, Zhang Y, Yuan ZH, Jiang C, et al. Transcriptome display during testicular differentiation of Channel catfish (Ictalurus punctatus) as revealed by RNA-seq analysis. Biol Reprod. (2016) 95:19. doi: 10.1095/biolreprod.116.138818

18. Du X, Wang B, Liu X, Liu X, He Y, Zhang Q, et al. Comparative transcriptome analysis of ovary and testis reveals potential sex-related genes and pathways in spotted knifejaw Oplegnathus punctatus. Gene. (2017) 637:203-10. doi: 10.1016/j.gene.2017.09.055

19. Wang Z, Qiu X, Kong D, Zhou X, Guo Z, Gao C, et al. Comparative RNA-Seq analysis of differentially expressed genes in the testis and ovary of Takifugu rubripes. Comp Biochem Physiol Part D Genomics Proteomics. (2017) 22:50-7. doi: 10.1016/j.cbd.2017.02.002

20. Presslauer C, Tilahun Bizuayehu T, Kopp M, Fernandes JM, Babiak I. Dynamics of miRNA transcriptome during gonadal development of zebrafish. Sentific Reports. (2017) 7:43850. doi: 10.1038/srep43850

21. Widmann C, Gibson S, Jarpe MB, Johnson GL. Mitogen-activated protein kinase: conservation of a three-kinase from yeast to human. Physiol Rev. (1999) 79:143-80. doi: 10.1152/physrev.1999.79.1.143

22. Chen S, Yang W, Zhang X, Jin J, Liang C, Wang J, et al. Melamine induces reproductive dysfunction via down-regulated the phosphorylation of $\mathrm{p} 38$ and downstream transcription factors Max and Sapla in mice testes. Sci Total Environ. (2021) 770:144727. doi: 10.1016/j.scitotenv.2020.144727

23. Jin J, Ma Y, Tong X, Yang W, Dai Y, Pan Y, et al. Metformin inhibits testosterone-induced endoplasmic reticulum stress in ovarian granulosa cells via inactivation of p38 MAPK. Hum Reprod. (2020) 35:114558. doi: 10.1093/humrep/deaa077

24. Deng Y, Zheng H, Yan Z, Liao D, Li C, Zhou J, et al. Full-length transcriptome survey and expression analysis of cassia obtusifolia to discover putative genes related to aurantio-obtusin biosynthesis, seed formation and development, and stress response. Int J Mol Sci. (2018) 19:2476. doi: 10.3390/ijms19092476

25. Westen AA, van der Gaag KJ, de Knijff P, Sijen T. Improved analysis of long STR amplicons from degraded single source and mixed DNA. Int J Legal Med. (2013) 127:741-7. doi: 10.1007/s00414-012-0816-1

26. Modi A, Vai S, Caramelli D, Lari M. The illumina sequencing protocol and the novaseq 6000 system. Methods Mol Biol. (2021) 2242:1542. doi: 10.1007/978-1-0716-1099-2_2

27. Hackl T, Hedrich R, Schultz J, Förster F. Proovread: large-scale high-accuracy PacBio correction through iterative short read consensus. Bioinformatics. (2014) 30:3004-11. doi: 10.1093/bioinformatics/btu392
28. Jia D, Wang Y, Liu Y, Hu J, Guo Y, Gao L, et al. SMRT sequencing of full-length transcriptome of flea beetle Agasicles hygrophila (Selman and Vogt). Sci Rep. (2018) 8:2197. doi: 10.1038/s41598-018-20181-y

29. Chin CS, Alexander DH, Marks P, Klammer AA, Drake J, Heiner C, et al. Nonhybrid, finished microbial genome assemblies from long-read SMRT sequencing data. Nat Methods. (2013) 10:563. doi: 10.1038/nmeth.2474

30. Kim D, Langmead B, Salzberg SL. HISAT: a fast spliced aligner with low memory requirements. Nat Methods. (2015) 12:357-60. doi: 10.1038/nmeth.3317

31. Robinson MD, McCarthy DJ, Smyth GK. edgeR: a Bioconductor package for differential expression analysis of digital gene expression data. Bioinformatics. (2010) 26:139-40. doi: 10.1093/bioinformatics/btp616

32. Leng N, Dawson JA, Thomson JA, Ruotti V, Rissman AI, Smits BM, et al. EBSeq: an empirical Bayes hierarchical model for inference in RNA-seq experiments. Bioinformatics. (2013) 29:2073. doi: 10.1093/bioinformatics/btt337

33. Altschul SF, Madden TL, Schäffer AA, Zhang J, Zhang Z, Miller W, et al. Gapped BLAST and PSI-BLAST: a new generation of protein database search programs. Nucleic Acids Res. (1997) 25:3389-402. doi: 10.1093/nar/25.17.3389

34. Young MD, Wakefeld MJ, Smyth GK, Oshlack A. Gene ontology analysis for RNA-seq: accounting for selection bias. Genome Biol. (2010) 11:R14. doi: 10.1186/gb-2010-11-2-r14

35. Wu J, Mao X, Cai T, Luo J, Wei L. KOBAS server: a web-based platform for automated annotation and pathway identification. Nucleic Acids Res. (2006) 34:W720-4. doi: 10.1093/nar/gkl167

36. Argyrous G. Statistics for Research: With a Guide to SPSS. London: SAGE. (2005).

37. D'Cotta H, Fostier A, Guiguen Y, Govoroun M, Baroiller JF. Aromatase plays a key role during normal and temperature-induced sex differentiation of tilapia oreochromis niloticus. Mol Reprod Dev. (2001) 59:265-76. doi: 10.1002/mrd.1031

38. Liu YX, Zhang Y, Li YY, Liu XM, Wang XX, Zhang CL, et al. Regulation of follicular development and differentiation by intra-ovarian factors and endocrine hormones. Front Biosci. (2019) 24:983-93. doi: 10.2741/4763

39. Shen $\mathrm{H}, \mathrm{Hu} \mathrm{Y}, \mathrm{Ma} \mathrm{Y}, \mathrm{Zhou} \mathrm{X}, \mathrm{Xu} \mathrm{Z}$, Shui Y, et al. In-depth transcriptome analysis of the red swamp crayfish Procambarus clarkii. PLoS ONE. (2014) 9:e110548. doi: 10.1371/journal.pone.0110548

40. Lin X, Zhou D, Zhang X, Li G, Zhang Y, Huang C, et al. A first insight into the gonad transcriptome of Hong Kong Catfish (Clarias fuscus). Animals. (2021) 11:1131. doi: 10.3390/ani11041131

41. Marisaldi L, Basili D, Gioacchini G, Canapa A, Carnevali O. De novo transcriptome assembly, functional annotation and characterization of the Atlantic bluefin tuna (Thunnus thynnus) larval stage. Mar Genomics. (2021) 58:100834. doi: 10.1016/j.margen.2020.100834

42. GuhaThakurta D. Computational identification of transcriptional regulatory elements in DNA sequence. Nucleic Acids Res. (2006) 34:3585-98. doi: 10.1093/nar/gkl372

43. Marra AN, Cheng CN, Adeeb B, Addiego A, Wesselman HM, Chambers $\mathrm{BE}$, et al. Iroquois transcription factor irx $2 \mathrm{a}$ is required for multiciliated and transporter cell fate decisions during zebrafish pronephros development. Sci Rep. (2019) 9:6454. doi: 10.1038/s41598-019-42943-y

44. Cioffi CC, Pollenz RS, Middleton DL, Wilson MR, Miller NW, William Clem L, et al. Oct2 transcription factor of a teleost fish: activation domains and function from an enhancer. Arch Biochem Biophys. (2002) 404:5561. doi: 10.1016/S0003-9861(02)00227-8

45. Jiang YH, Han KH, Wang SH, Chen Y, Wang YL, Zhang ZP. Identification and expression of transcription factor sox 2 in large yellow croaker Larimichthys crocea. Theriogenology. (2018) 120:123-37. doi: 10.1016/j.theriogenology.2018.07.025

46. Yao F, Liu Y, Du L, Wang X, Zhang A, Wei H, et al. Molecular identification of transcription factor Runxl variants in grass carp (Ctenopharyngodon idella) and their responses to immune stimuli. Vet Immunol Immunopathol. (2014) 160:201-8. doi: 10.1016/j.vetimm.2014.05.002

47. Schmitges FW, Radovani E, Najafabadi HS, Barazandeh M, Campitelli LF, Yin $\mathrm{Y}$, et al. Multiparameter functional diversity of human $\mathrm{C} 2 \mathrm{H} 2$ zinc finger proteins. Genome Res. (2016) 26:1742-52. doi: 10.1101/gr.209643.116 
48. Lee SU, Maeda T. POK/ZBTB proteins: an emerging family of proteins that regulate lymphoid development and function. Immunol Rev. (2012) 247:10719. doi: 10.1111/j.1600-065X.2012.01116.x

49. Wu X, Yang Y, Zhong C, Guo Y, Li S, Lin H, et al. Transcriptome profiling of laser-captured germ cells and functional characterization of zbtb40 during 17alpha - methyltestosterone - induced spermatogenesis in orange-spotted grouper (Epinephelus coioides). BMC Genomics. (2020) 21:113. doi: $10.1186 / \mathrm{s} 12864-020-6477-4$

50. Buaas FW, Kirsh AL, Sharma M, McLean DJ, Morris JL, Griswold MD, et al. $\mathrm{Plzf}$ is required in adult male germ cells for stem cell selfrenewal. Nat Genet. (2004) 36:647-52. doi: 10.1038/ng1366

51. Lovelace DL, Gao Z, Mutoji K, Song YC, Ruan J, Hermann BP. The regulatory repertoire of PLZF and SALL4 in undifferentiated spermatogonia. Development. (2016) 143:1893-906. doi: 10.1242/dev. 132761

52. Van Muiswinkel WB, Lamers CH, Rombout JH. Structural and functional aspects of the spleen in bony fish. Res Immunol. (1991) 142:3626. doi: 10.1016/0923-2494(91)90093-X

53. Bates T, Naumann U, Hoppe B, Englert C. Kidney regeneration in fish. Int J Dev Biol. (2018) 62:419-29. doi: 10.1387/ijdb.170335ce

54. Geven EJW, Klaren PHM. The teleost head kidney: integrating thyroid and immune signalling. Dev Comp Immunol. (2017) 66:73-83. doi: 10.1016/j.dci.2016.06.025

55. Zhang $\mathrm{Z}$, Chi $\mathrm{H}$, Dalmo RA. Trained innate immunity of fish is a viable approach in larval aquaculture. Front Immunol. (2019) 10:42. doi: 10.3389/fimmu.2019.00042

56. Cowan M, Azpeleta C, López-Olmeda JF. Rhythms in the endocrine system of fish: a review. J Comp Physiol B. (2017) 187:1057-89. doi: 10.1007/s00360-017-1094-5

57. Reinecke M. Insulin-like growth factors and fish reproduction. Biol Reprod. (2010) 82:656-61. doi: 10.1095/biolreprod.109.080093

58. Dupont J, Scaramuzzi RJ. Insulin signalling and glucose transport in the ovary and ovarian function during the ovarian cycle. Biochem J. (2016) 473:1483e1501. doi: 10.1042/BCJ20160124

59. Otani T, Maruo T, Yukimura N, Mochizuki M. Effect of insulin on porcine granulosa cells: implications of a possible receptor mediated action. Acta Endocrinol. (1985) 108:104-10. doi: 10.1530/acta.0.10 80104

60. Samoto T, Maruo T, Ladines-Llave CA, Matsuo H, Deguchi JU, Barnea ER, et al. Insulin receptor expression in follicular and stromal compartments of the human ovary over the course of follicular growth, regression and atresia. Endocr J. (1993) 40:715-26. doi: 10.1507/endocrj. 40.715

61. Bossaert P, De Cock H, Leroy JLMR, De Campeneere S, Bols PEJ, Filliers M, et al. Immunohistochemical visualization of insulin receptors in formalinfixed bovine ovaries post mortem and in granulosa cells collected in vivo. Theriogenology. (2010) 73:1210-9. doi: 10.1016/j.theriogenology.2010. 01.012

62. Zheng W, Nagaraju G, Liu Z, Liu K. Functional roles of the phosphatidylinositol 3-kinases (PI3Ks) signaling in the mammalian ovary. Mol Cell Endocrinol. (2012) 356:24-30. doi: 10.1016/j.mce.2011.05.027

63. Bogacka I, Kurzynska A, Bogacki M, Chojnowska K. Peroxisome proliferatoractivated receptors in the regulation of female reproductive functions. Folia Histochemica Cytobiologica. (2015) 53:189-200. doi: 10.5603/fhc.a2015. 0023

64. Barker HM, Brewis ND, Street AJ, Spurr NK, Cohen PT. Three genes for protein phosphatase 1 map to different human chromosomes: sequence, expression and gene localisation of protein serine/threonine phosphatase 1 beta (PPP1CB). Biochim Biophys Acta. (1994) 1220:2128. doi: 10.1016/0167-4889(94)90138-4

65. Downs SM, Hudson ER, Hardie DG. A potential role for AMP-activated protein kinase in meiotic induction in mouse oocytes. Dev Biol. (2002) 245:200-12. doi: 10.1006/dbio.2002.0613

66. Dupont J, Reverchon M, Cloix L, Froment P, Rame C. Involvement of adipokines,AMPK, PI3K and the PPAR signaling pathways in ovarian follicle development and cancer. Int J Develop Biol. (2012) 56:95967. doi: $10.1387 / \mathrm{ijdb} .120134 \mathrm{jd}$
67. Reverchon M, Cornuau M, Rame C, Guerif F, Royere D, Dupont J. Chemerin inhibits IGF-1-induced progesterone and es- tradiol secretion in human granulosa cells. Human Reproduction. (2012) 27:1790-800. doi: 10.1093/humrep/des089

68. Chappaz E, Albornoz MS, Campos D, Che L, Palin MF, Murphy BD, et al. Adiponectin enhances in vitro development of swine embryos. Domest Anim Endocrinol. (2008) 35:198-207. doi: 10.1016/j.domaniend.2008. 05.007

69. Young PR, McLaughlin MM, Kumar S, Kassis S, Doyle ML, McNulty D, et al Pyridinyl imidazole inhibitors of p38 mitogen-activated protein kinase bind in the ATP site. J Biol Chem. (1997) 272:12116-21. doi: 10.1074/jbc.272.18. 12116

70. Zhang Y, Zhang K, Zhang Y, Zhou L, Huang H, Wang J. IL18 Mediates vascular calcification induced by high-fat diet in rats with chronic renal failure. Front Cardiovasc Med. (2021) 25:724233. doi: 10.3389/fcvm.2021.724233

71. Lu ZY, Fan J, Yu LH, Ma B, Cheng LM. The Up-regulation of TNF- $\alpha$ maintains trigeminal neuralgia by modulating MAPKs phosphorylation and BKCa channels in trigeminal nucleus caudalis. Front Cell Neurosci. (2021) 15:764141. doi: 10.3389/fncel.2021. 764141

72. O’Neil MM, Korthanke CM, Scarpa JO, Welsh TH, Cardoso RC, Williams GL. Differential regulation of gonadotropins in response to continuous infusion of native gonadotropin-releasing hormone in the winter anovulatory mare and effects of treatment with estradiol17ß. J Equine Vet Sci. (2019) 75:93-103. doi: 10.1016/j.jevs.2019. 01.013

73. Scarmuzzi RJ, Brown HM, Dupont J. Nutritional and metabolic mechanisms in the ovary and their role in mediating the effects of diet on folliculogenesis: a perspective. Reprod Domestic Animals. (2010) 45:3241. doi: 10.1111/j.1439-0531.2010.01662.x

74. Ortmann O, Weiss JM, Diedrich K. Gonadotrophin-releasing hormone $(\mathrm{GnRH})$ and GnRH agonists: mechanisms of action. Reprod Biomed Online. (2002) 5:1-7. doi: 10.1016/S1472-6483(11)60210-1

75. Breton B, Govoroun M, Mikolajczyk T. GTH I and GTH II secretion profiles during the reproductive cycle in female rainbow trout: relationship with pituitary responsiveness to GnRH-A stimulation. Gen Comp Endocrinol. (1998) 111:38-50. doi: 10.1006/gcen.1998.7088

76. Hildahl J, Sandvik GK, Edvardsen RB, Fagernes C, Norberg B, Haug TM, et al. Identification and gene expression analysis of three GnRH genes in female Atlantic cod during puberty provides insight into $\mathrm{GnRH}$ variant gene loss in fish. Gen Comp Endocrinol. (2011) 172:458-67. doi: 10.1016/j.ygcen.2011. 04.010

77. Burow S, Fontaine R, von Krogh K, Mayer I, Nourizadeh-Lillabadi R, Hollander-Cohen L, et al. Medaka follicle-stimulating hormone (Fsh) and luteinizing hormone (Lh): Developmental profiles of pituitary protein and gene expression levels. Gen Comp Endocrinol. (2019) 272:93-108. doi: 10.1016/j.ygcen.2018.12.006

78. Hollander-Cohen L, Golan M, Levavi-Sivan B. Differential regulation of gonadotropins as revealed by transcriptomes of distinct LH and FSH cells of fish pituitary. Int J Mol Sci. (2021) 22:6478. doi: 10.3390/ijms22126478

79. Waszkiewicz EM, Zmijewska A, Kozlowska W, Franczak A. Effects of LH and FSH on androgen and oestrogen release in the myometrium of pigs during the oestrous cycle and early pregnancy. Reprod Fertil Dev. (2020) 32:1200-11. doi: 10.1071/RD20148

80. Nelson LE, Sheridan MA. Regulation of somatostatins and their receptors in fish. Gen Comp Endocrinol. (2005) 142:11733. doi: 10.1016/j.ygcen.2004.12.002

81. Gonzalez-Robayna IJ, Falender AE, Ochsner S, Firestone GL, Richards JS. Follicle- stimulating hormone (FSH) stimulates phosphorylation and activation of protein kinase $\mathrm{B}$ (PKB/Akt) and serum and glucocorticoid- induced kinase (Sgk): evidence for a kinase-independent signaling by FSH in granulosa cells. Molec Endocrinol. (2000) 14:1283-300. doi: 10.1210/mend.14.8.0500

82. Li M, Sun L, Wang D. Roles of estrogens in fish sexual plasticity and sex differentiation. Gen Comp Endocrinol. (2019) 277:9-16. doi: 10.1016/j.ygcen.2018.11.015 
83. Ström JO, Theodorsson A, Ingberg E, Isaksson IM, Theodorsson E. Ovariectomy and $17 \beta$-estradiol replacement in rats and mice: a visual demonstration. J Vis Exp. (2012) 7:e4013. doi: 10.3791/4013

84. Almeida-Pereira G, Vilhena-Franco T, Coletti R, Cognuck SQ, Silva HVP, Elias LLK, et al. 17 $\beta$-Estradiol attenuates p38MAPK activity but not PKC $\alpha$ induced by angiotensin II in the brain. J Endocrinol. (2019) 240:34560. doi: 10.1530/JOE-18-0095

Conflict of Interest: CL was employed by the company Xinjiang Tianyun Organic Agriculture Co., Yili Group.

The remaining authors declare that the research was conducted in the absence of any commercial or financial relationships that could be construed as a potential conflict of interest.
Publisher's Note: All claims expressed in this article are solely those of the authors and do not necessarily represent those of their affiliated organizations, or those of the publisher, the editors and the reviewers. Any product that may be evaluated in this article, or claim that may be made by its manufacturer, is not guaranteed or endorsed by the publisher.

Copyright (๑) 2022 Huang, Gu, Liu, Zhang, Dong, He, Jiao, Li, Wang and Xu. This is an open-access article distributed under the terms of the Creative Commons Attribution License (CC BY). The use, distribution or reproduction in other forums is permitted, provided the original author(s) and the copyright owner $(s)$ are credited and that the original publication in this journal is cited, in accordance with accepted academic practice. No use, distribution or reproduction is permitted which does not comply with these terms. 